\title{
Emotional oddball: A review on memory effects
}

\author{
Helge Schlüter ${ }^{1} \cdot$ Ryan P. Hackländer ${ }^{1}$. Christina Bermeitinger ${ }^{1}$
}

Published online: 20 August 2019

(C) The Psychonomic Society, Inc. 2019

\begin{abstract}
How do emotional stimuli influence perception, attention, and ultimately memory? This debate at the cross-section of emotion and cognition research has a long tradition. The emotional oddball paradigm (EOP) has frequently been applied to investigate the detection and processing of (emotional) change detection (Schlüter \& Bermeitinger, 2017). However, the EOP has also been used to reveal the effects of emotional deviants on memory for serially presented stimuli. In this integrative article, we review the results of 29 experiments published between the years 2000 and 2017. Based on these data, we provide an overview of how the EOP is applied in the context of memory research. We also review and integrate the empirical evidence for memory effects in the EOP (with a special focus on retrograde and anterograde emotion-induced effects) and present theories of emotional memory as well as their fit with the results obtained by the EOP. Directions for future research are presented that would help to address important issues of the current debate around emotion-induced memory effects.
\end{abstract}

Keywords Emotional oddball $\cdot$ Emotional stimuli $\cdot$ Memory $\cdot$ Deviation $\cdot$ Expectation

\section{Introduction}

Imagine taking part in The Generation Game, a British game show that had become very popular during the late 1970s. At the end of the game show, contestants faced a conveyor belt, and their task was to memorize the objects (or prizes) running on the belt. Now imagine sitting in front of that belt trying to memorize the prizes that are presented one after the other: a basket with bread, gardening tools, a whiskey bottle, an old table lamp, a picnic flask, a gruesome Halloween mask, a backgammon set, and an electric wall clock. In this situation, it would be most likely that your attention was especially drawn by the Halloween mask. Studies have shown that items sticking out of the mass are detected fast and automatically draw attention (Wolfe, 2003). Therefore, it is not surprising that you, as a contestant, would most likely recall the Halloween mask (i.e., the deviant stimulus) after the presentation of all prizes. But what about the other items, especially those that were presented directly before and after the mask on the

Helge Schlüter

helge.schlueter@uni-hildesheim.de

1 Department of Psychology, Experimental Psychology, University of Hildesheim, Universitätsplatz 1, D-31141 Hildesheim, Germany conveyor belt? Is memory for those items somehow affected by the presentation of a deviant item?

In the lab, these questions can be tackled by the oddball paradigm, which has repeatedly been used to explore the underlying cognitive and neurophysiological components of change detection and attentional processes. It has also been used to investigate the special role of emotional content in perception and attention (emotional oddball paradigm; EOP). In the EOP (as in the oddball paradigm), there are typically two types of stimuli: standards (which are presented in a large majority of trials, generally $80 \%$ ) and deviants (for a review on the EOP, see Schlüter \& Bermeitinger, 2017). The EOP is used as a tool to address questions such as: (a) to what degree do emotional deviant stimuli trigger orientation reactions, (b) which role does the emotional context play in the processing of deviant information, and (c) how does the processing of emotional deviant information differ inter-individually? Additionally, a substantial number of studies utilize the EOP to investigate the influence of emotional stimuli on memory processes. The current review is specifically concerned with this question.

The emotional valence of a stimulus or an event can be considered a marker of either appetitive or aversive nature for events, which might have relevance for the survival and the fitness of an individual. Both positive and negative emotional content can enhance (episodic) memory in humans (Adelman \& Estes, 2013). This mechanism is, from an 
evolutionary perspective, highly adaptive, in that it ensures that important information is available for future use (Hamann, 2001).

Studying memory effects of stimuli that stick out of the mass and differ from the general context has a long tradition (e.g., Saufley \& Winograd, 1970; Schultz, 1971; Tulving, 1969). In these early studies, participants were presented with sequences of common words (standards) and names of celebrities (deviants). Memory was especially poor for common words directly preceding a celebrity name. This effect is known as "induced retrograde amnesia."

Later, retrograde amnesia (i.e., impaired memory for items preceding a deviant stimulus) and anterograde amnesia (i.e., impaired memory for items following a deviant stimulus) were found in cases of high arousal that was induced by the presentation of pictures of nudes in the middle position of a sequence (Detterman \& Ellis, 1972; Ellis, Detterman, Runcie, McCarver, \& Craig, 1971; Runcie \& O'Bannon, 1977). Detterman (1976) was able to show amnesic effects of arousing deviants (loud shouts as compared to spoken words in a normal volume) on the recall of spoken words in a normal volume surrounding the deviants.

Though some of these early studies provided first hints that arousing stimuli impair memory for temporally adjacent events, they lacked a control condition that would have allowed one to conclude that the anterograde and retrograde effects were really due to the arousal induction by the deviant stimuli used. For instance, Ellis et al. (1971) compared free-recall rates (Exp. 1) as well as recognition rates (Exp. 2) for experimental sequences that included an arousing picture of a nude in the middle position with the respective memory rates for stimuli of sequences that did not include a deviant. However, in order to investigate arousal-induced amnesic effects, the control condition should have contained an emotionally neutral, low arousal, deviant.

From the beginning of this century (i.e., in the year 2000), the first experiments evolved that we would term EOP experiments in a narrow sense. Whereas in earlier experiments there was no comparison between control conditions that included neutral deviants and experimental conditions that included emotional deviants, the more recent experiments now involved this comparison (e.g., Strange, Henson, Friston, \& Dolan, 2000; Strange \& Dolan, 2001; Strange, Hurlemann, \& Dolan, 2003). The inclusion of an emotional control condition is a crucial element if one is interested in the effect of emotional stimuli on the remembrance of neighboring neutral information. These emotional memory effects were examined more systematically in the EOP experiments by varying encoding task demands, the levels of arousal and valence, and the semantic relatedness of the stimuli. Also, withinsubjects designs have been employed and the serial position of deviants has usually been varied in a pseudorandom manner. The study by Strange et al. (2000) represented the beginning of a series of experiments on the differential influences of emotional deviant stimuli on the memory performances for preceding and subsequent neutral standard stimuli.

The most common way to investigate memory via the EOP is through a design with several trials. Each trial involves an encoding phase, in which a certain number of stimuli are presented sequentially (a classification task is most often demanded at encoding), and a retrieval phase, during which the participants are required to remember the stimuli of the encoding phase. Each of the sequences contains one or more deviants. The critical memory rates then are the recall/recognition of deviants as well as the recall/recognition of the neutral stimuli directly surrounding the deviants (see Fig. 1a for a depiction of anterograde and retrograde memory effects in the EOP).

To our knowledge, no attempt has yet been made to review evidence from the recent EOP experiments on memory. At first glance, the results seem to be rather incoherent, with emotionally negative (highly arousing) deviant stimuli sometimes impairing memory rates of their preceding neighbors (e.g., Müller, Wohlrath, Kopp, \& Lengler, 2009) and sometimes enhancing them (e.g., Sakaki, Fryer, \& Mather, 2014). The EOP experiments reviewed in this article differ largely in how memory was assessed, which needs to be addressed. Also, it is important to highlight the role of emotional valence since some researchers have found that negative deviants

Fig. 1 a Outline of memory effects in the EOP. Sequentially presented emotionally neutral standard stimuli (S) are interrupted by deviants. Deviant stimuli are either emotionally positive (Pos), neutral (Neu), or negative (Neg). Neutral deviants differ only semantically or only perceptually from neutral standards. Note that most identified EOP studies assign position and type of deviant in a pseudorandom manner. Emotion-induced memory effects can be observed for standard stimuli directly preceding an emotional deviant (position -1) and/or for standards directly following an emotional deviant (position +1 ). The former is referred to as a retrograde effect, the latter as an anterograde effect of the deviant on the memory performance for neighboring standard information b This figure is a schematic depiction of a typical experimental structure of an EOP with words. In 14 EOP experiments the stimuli were exclusively words, and in five experiments words were used as standards and human faces were used as deviants. Most of these experiments employ multi-oddball sequences, with a median number of 19 stimuli per sequence (ranging from eight to 480 stimuli). Median SOA and stimulus presentation time (PT) in these experiments were $3,000 \mathrm{~ms}$ $\left(M_{\mathrm{SOA}}=3,226 \mathrm{~ms}, S D_{\mathrm{SOA}}=777 \mathrm{~ms}\right)$ and $1,000 \mathrm{~ms}\left(M_{\mathrm{PT}}=1,074 \mathrm{~ms}\right.$, $S D_{\mathrm{PT}}=529 \mathrm{~ms}$ ), respectively c Schematic depiction of a typical trial structure of the ten EOP experiments with picture-label pairs. All of these experiments employed single-oddball sequences, with a median number of eight stimuli per sequence (ranging from five to ten). Median SOA and PT in these experiments were $3,000 \mathrm{~ms}\left(M_{\mathrm{SOA}}=\right.$ $\left.3,170 \mathrm{~ms}, S D_{\mathrm{SOA}}=1,713 \mathrm{~ms}\right)$ and $2,000 \mathrm{~ms}\left(M_{\mathrm{PT}}=1,670 \mathrm{~ms}, S D_{\mathrm{PT}}=\right.$ $447 \mathrm{~ms}$ ), respectively 
a

Effect: retrograde

effect of

deviant

anterograde

effect of

deviant

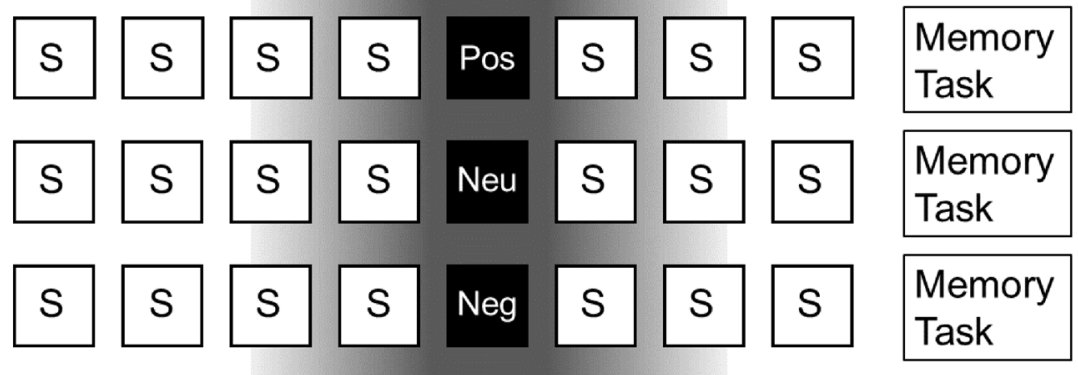

Position:

-1 deviant +1
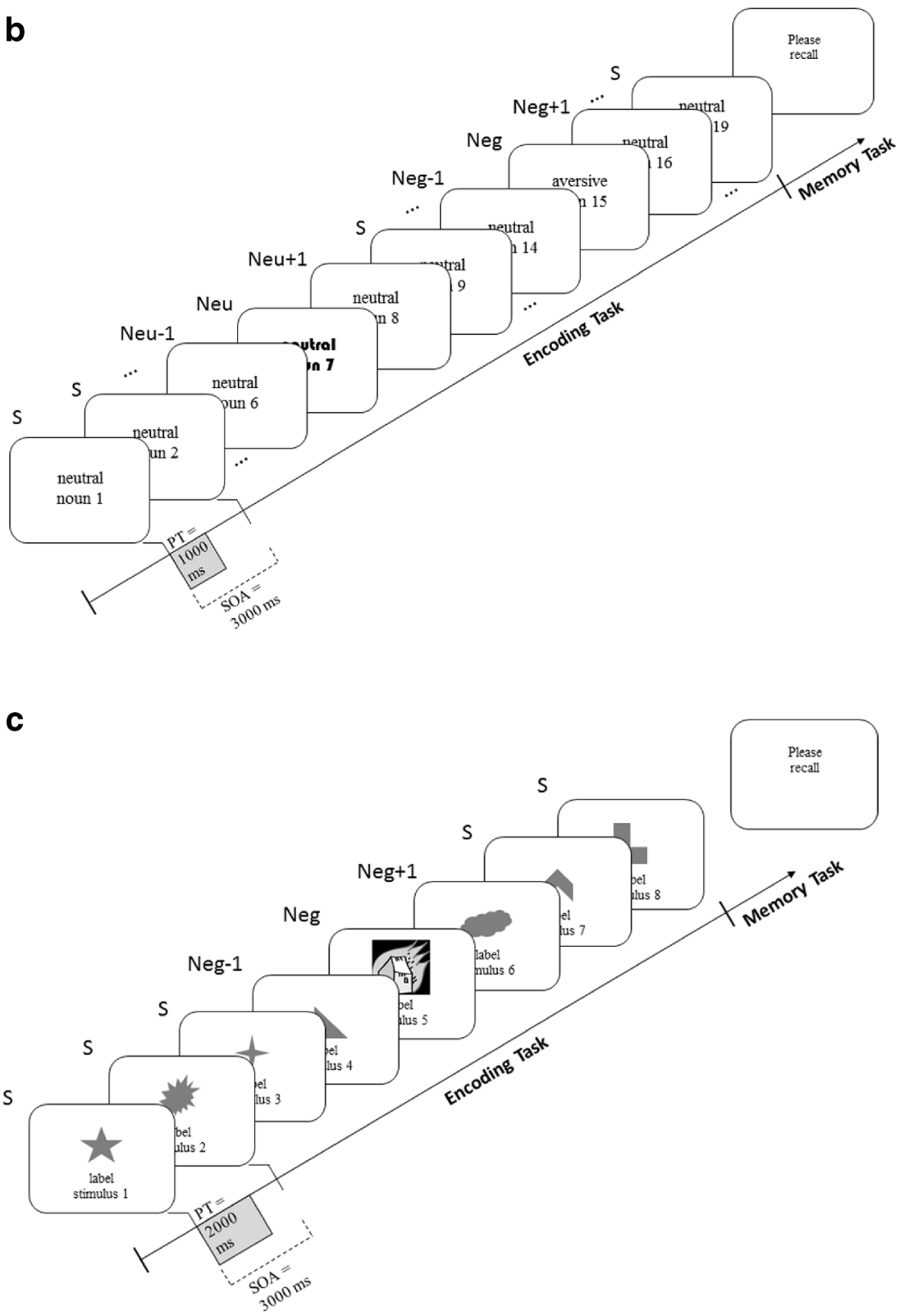
impair, but positive deviants enhance, memory for preceding stimuli (e.g., Hurlemann et al., 2005). The influence of positive deviant stimuli, however, seems to have been slightly disregarded in the current debate about emotion-induced memory effects (e.g., Mather \& Sutherland, 2011).

Thus, it is important to review the behavioral results obtained via EOPs to help determine what factors moderate/mediate how emotion influences memory for surrounding information. As more recent studies allow for stronger conclusions due to their methodological advantages (see above), we only included studies published in or after the year 2000. In this review, we aimed (1) to provide an exhaustive overview of recent literature regarding memory effects in EOP experiments, (2) to systematically review and integrate empirical results, and (3) to discuss theoretical explanations and implications, as well as to inspire future research on this topic.

\section{Sample description}

Twenty-nine recent EOP experiments (taken from 19 publications), published between 2000 and 2017, involve a memory assessment after presentation of a sequence of stimuli. Noteworthy, it is not memory for the deviant stimulus itself that is most often of highest interest for the researchers, but rather memory for the (mostly) emotionally neutral stimuli preceding and following the deviant stimulus in the encoding stage. We only included EOP experiments in our detailed analysis that employed memory tasks and that involved a comparison between emotional and non-emotional deviants, which led to the exclusion of seven studies (no non-emotional control condition: Richardson, Strange, \& Dolan, 2004a; Richardson, Strange, Duncan, \& Dolan, 2003, 2006; Richardson, Strange, Thompson, Baxendale, Duncan, \& Dolan, 2004b; Schmidt, 2002; no report of behavioral/ memory data: Strange \& Dolan, 2001, 2007).

Twenty-three out of 29 of the reviewed EOP experiments included an encoding task. Of these, all experiments with pictorial stimuli required a classification task in the encoding stage that involved deep encoding ( $n=6$; e.g., "Is the presented picture natural or artificial?"). Also, the majority of EOP experiments with words employed classification tasks, with more shallow ( $n=9$; e.g., "Does the first letter of the presented word have an enclosed space?") than deep encoding tasks ( $n=$ $5)$. Four experiments with words employed both types of tasks, while one experiment employed a shallow deviant detection task instead of a classification task during encoding. Four EOP experiments with pictures and two with words did not demand any specific task (related with a button-press) during the encoding stage other than to memorize each stimulus.
The identified experiments employed one or two of three types of memory tasks. When facing free-recall tasks, participants are prompted to recall certain events or stimuli without the presentation of any kind of cue that might facilitate recollection. By contrast, in cued recall tasks, in addition to the prompt, a cue is provided. This might be, for example, the first letter of a word, which helps to recollect the target item. Recognition tasks provide the strongest cues by presenting stimuli that might involve the original stimulus itself. The participants' task now is to decide whether the given stimulus was presented before or not. Recognition tasks may involve the exact same stimuli, similar stimuli, and stimuli that had not been presented during encoding. Presenting these three classes of stimuli during the recognition stage allows for the calculation of recognition rates in different ways, either assessing specific recognition (i.e., did the participant recollect the item in detail?) or general recognition (i.e., did the participant recollect the general theme - also called gist information - of an item but not the exact same item?). The most common memory task in the 29 identified EOP experiments was the free-recall task $(n=16)$, followed by recognition tasks $(n=9)$ and cued recall tasks $(n=8$; note that in two experiments both recall and recognition tasks were used, while one experiment involved a free and a cued recall task).

The memory tasks used in the identified EOP literature also differ with regard to the time at which memory was assessed and how the material was learned. Table 1 provides an overview of the number of immediate and delayed-memory tasks and the number of instructed and incidental tasks.

In the analyzed experiments, the median number of stimuli per sequence is 14 (range: 5-480). The time between the onsets of two meaningful stimuli in a sequence is often called stimulus-onset asynchrony (SOA). Mean SOA in the reviewed experiments is about $3 \mathrm{~s}(M=3,206 \mathrm{~ms} ; S D=$ $1,178 \mathrm{~ms}$; range: $1,000-5,000 \mathrm{~ms})$, while stimulus presentation time is about $1 \mathrm{~s}$ on average $(M=1,294 \mathrm{~ms} ; S D=572 \mathrm{~ms}$; range: $250-3,000 \mathrm{~ms}$ ). Please see Figs. $1 \mathrm{~b}$ and 1c for more detailed information on experimental procedures.

Critical for concluding whether emotional deviant stimuli led to amnesic or hypermnesic effects on neighboring stimuli is to determine the baseline to which memory performance for items surrounding the deviants is compared. One way to calculate a baseline for comparison is to randomly draw a number of neutral standards (usually two) from each trial and use the average memory performance of all drawn standard stimuli as a means to express memory performance for neutral standard information (we will refer to this as the "compare with standards method"). Thus, retrograde and anterograde memory effects are 
Table 1 Overview of memory tasks in the identified EOP experiments

\begin{tabular}{|c|c|c|c|c|c|}
\hline time of assessment & type of assessment & incidental & instructed/prepared & not reported & $n$ (type of task) \\
\hline \multirow[t]{3}{*}{$\begin{array}{l}\text { immediate after } \\
\text { each trial }\end{array}$} & free recall & & $\begin{array}{l}16 \text { experiments (Hurlemann et al., 2005, } \\
\text { Exp. 1, 2; } \\
\text { Hurlemann et al., 2007a, 2007b; } \\
\text { Kamp et al., 2015; } \\
\text { Knight \& Mather, 2009; Exp. 1, 2; } \\
\text { Miu et al., 2005, condition 1; } \\
\text { Smith \& Beversdorf, 2008; } \\
\text { Strange et al., 2000, 2003, Exp. 1, 2, 3; } \\
\text { Strange et al., 2008, 2014; } \\
\text { West Saxvig et al., 2008) }\end{array}$ & & 16 \\
\hline & cued recall & & 1 (Miu et al., 2005, condition 2) & & 1 \\
\hline & recognition & & 1 (Müller et al., 2009) & & 1 \\
\hline $\begin{array}{l}\text { immediate after } \\
\text { each block }\end{array}$ & recognition & & $\begin{array}{l}2 \text { (Clewett et al., 2017; } \\
\quad \text { Froeliger et al., 2009) }\end{array}$ & & 2 \\
\hline \multirow[t]{2}{*}{$\begin{array}{l}\text { immediate at the } \\
\text { end of session }\end{array}$} & cued recall & & & 1 (Strange et al., 2010; Exp. 2) & 1 \\
\hline & recognition & & 3 (Sakaki et al., 2014, Exp. 1, 2, 3) & 1 (Strange \& Dolan, 2004) & 4 \\
\hline \multirow[t]{2}{*}{ delayed } & cued recall & $\begin{array}{l}1 \text { (Miu et al., } 2005 \text {, } \\
\text { condition } 3 \text {; } \\
\text { delay: } 1 \text { day) }\end{array}$ & & $\begin{array}{l}5 \text { (Strange et al., 2010; Exp. 1, 3, 4, } 5 \\
\quad \text { delays: } 1 \text { day }-7 \text { days; } \\
\text { Kroes et al., 2010; delays: } 1-2 \text { days) }\end{array}$ & 6 \\
\hline & recognition & & & $\begin{array}{l}2 \text { (Knight \& Mather, 2009, Exp. 1, 2; } \\
\quad \text { delay: } 7 \text { days) }\end{array}$ & 2 \\
\hline$n$ (type of instruction) & & 1 & 23 & 9 & 33 \\
\hline
\end{tabular}

reported as deviations from standard recall/recognition (Miu, Heilman, Opre, \& Miclea, 2005; Müller et al., 2009; Smith \& Beversdorf, 2008; Strange et al., 2000, 2003; Strange \& Dolan, 2004; Strange, Kroes, Roiser, Tan, \& Dolan, 2008; West Saxvig, Johansen Lundervold, Gronli, Ursin, Bjorvatn, \& Portas, 2008). Note that some authors who used this strategy acknowledged possible primacy effects by excluding the first lot of neutral standards of a sequence from analysis (e.g., Müller et al., 2009; Strange et al., 2003, 2004, 2008), while others did not (e.g., Smith \& Beversdorf, 2008).

Another common method of comparison is to directly contrast trials that include emotionally neutral deviants (i.e., perceptual or semantic deviants) with emotionally positive/ negative deviant trials (we will refer to this as the "contrastive method"). This way, memory performances for neutral deviants as well as for the neutral standards directly preceding and following the neutral deviants serve as a baseline (Clewett, Sakaki, Nielsen, Petzinger, \& Mather, 2017; Hurlemann et al., 2005, 2007a, 2007b, Knight \& Mather, 2009; Sakaki et al., 2014, Strange et al., 2010).

We illustrate retrograde (i.e., from a deviant to the preceding standard) and anterograde (i.e., from the deviant to the following standard) memory effects in experiments that employed immediate-recall tasks using the "compare with standards method" in Fig. 2 and the "contrastive method" in Fig. 3. In addition, these figures provide information about the magnitude of deviant recall. The figures will be discussed in detail in the summary section.

\section{The (emotional) oddball effect}

A typical finding - though not the main focus of this review is an enhanced memory rate for all types of deviant stimuli (we will refer to this as the oddball effect). This mnemonic enhancement for the exceptional over the mundane in a list of otherwise homogenous stimuli is also known as the von Restorff effect (von Restorff, 1933; Wallace, 1965).

There is, however, evidence that the oddball effect is not always found. For example, a recent EOP study with words found that only negative (and not positive) deviant words were recalled more than standard stimuli (Kamp, Potts, \& Donchin, 2015). The fact that the oddball effect is not always found fits with the rest of memory research, which has failed to determine any general laws of the nature of remembering (Roediger III, 2008). Although the (emotional) oddball effect is interesting in and of itself, the focus of the majority of the identified EOP experiments lies on the effects of deviant stimuli on the memory rate for surrounding items rather than memory rates for the deviants themselves. For an overview of oddball effects as well as other memory effects in the reviewed EOP studies see Table 2.

\section{Memory effects of emotional deviant stimuli on neighboring standard stimuli}

In the following section, we give a comprehensive overview of behavioral evidence for emotion-induced memory effects from the relevant EOP studies. An additional section is 


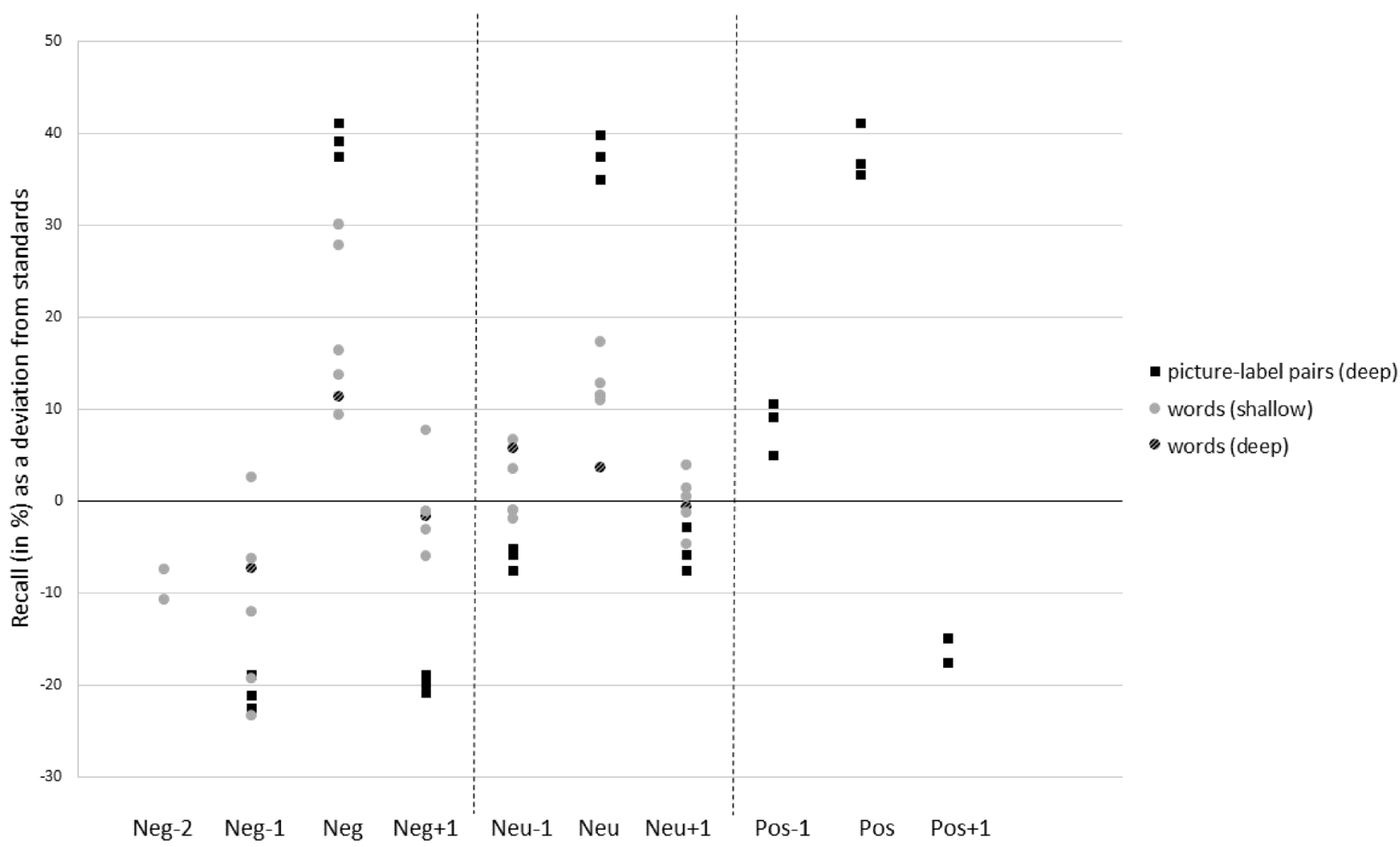

Fig. 2 Deviations of averaged recall rates for all investigated stimulus positions as compared with the averaged recall rates for standard stimuli (in percent) from the respective EOP experiment (i.e., "compare with standards method"). Included are only EOP experiments that involve an immediate free-recall task. Data for picture-label pairs are derived from Hurlemann et al. (2005: Exp. 1; 2007a: healthy controls; 2007b: healthy controls), data for words are derived from Strange et al. (2003: Exp. 1, split in shallow and deep encoding, Exp. 2 and Exp. 3) and Strange et al. (2008: split for genotypes investigated). While all experiments using picture-label pairs employed deep encoding tasks (black quadratic figures), light gray round figures indicate that the word EOP used a

dedicated to physiological and pharmacological effects observed in some of the identified studies. We provide a detailed description of emotion-induced memory effects in the EOP and highlight three main factors (emotionality, semantic relatedness, and goal relevance) that were investigated by researchers using the paradigm. Most of this research involves immediate memory tasks (that we define as taking place in the same session as the encoding tasks), but some use delayedmemory tasks to answer specific questions, as to how the influence of emotional deviants on memory rates of surrounding information develops or changes if the memory task is delayed by a day or even up to a week. Since delayed tasks were not included to investigate the EOP but rather to answer other specific research questions, we decided to report evidence sorted only by the three main factors. However, we include the more technical factor of memory task delay in the summary section. shallow encoding task and the dark gray round figures stand for an experiment with words and a deep encoding task.

Please note that exact values were only available for Hurlemann et al. (2007a). Other values had to be estimated from diagrams. Data from Miu et al. (2005) are not included because no differentiation was made between positive and negative deviants. Positive values indicate hypermnesic effects (i.e. an enhanced recall rate for the respective position compared with the average neutral standard recall of the respective experiment), negative values indicate amnesic effects (i.e., a decreased recall rate)

\section{Evidence for emotion-induced memory effects}

The influence of emotional valence/arousal The first key factor is the emotionality of the deviant itself with a focus on emotional arousal or valence or both. There has been a long debate over which properties of emotional stimuli are more influential in memory. While a large body of evidence is concerned with the impact of arousal on the probability to remember an item (e.g., Mather \& Sutherland, 2011), other research has highlighted the importance of valence, with pleasant (positive) and unpleasant (negative) stimuli leading to different results with regard to memory performances (e.g., Kensinger, 2009). The EOP experiments in this subsection provide evidence for arousal- as well as valence-induced effects of deviants on the memory rate for surrounding neutral information. 


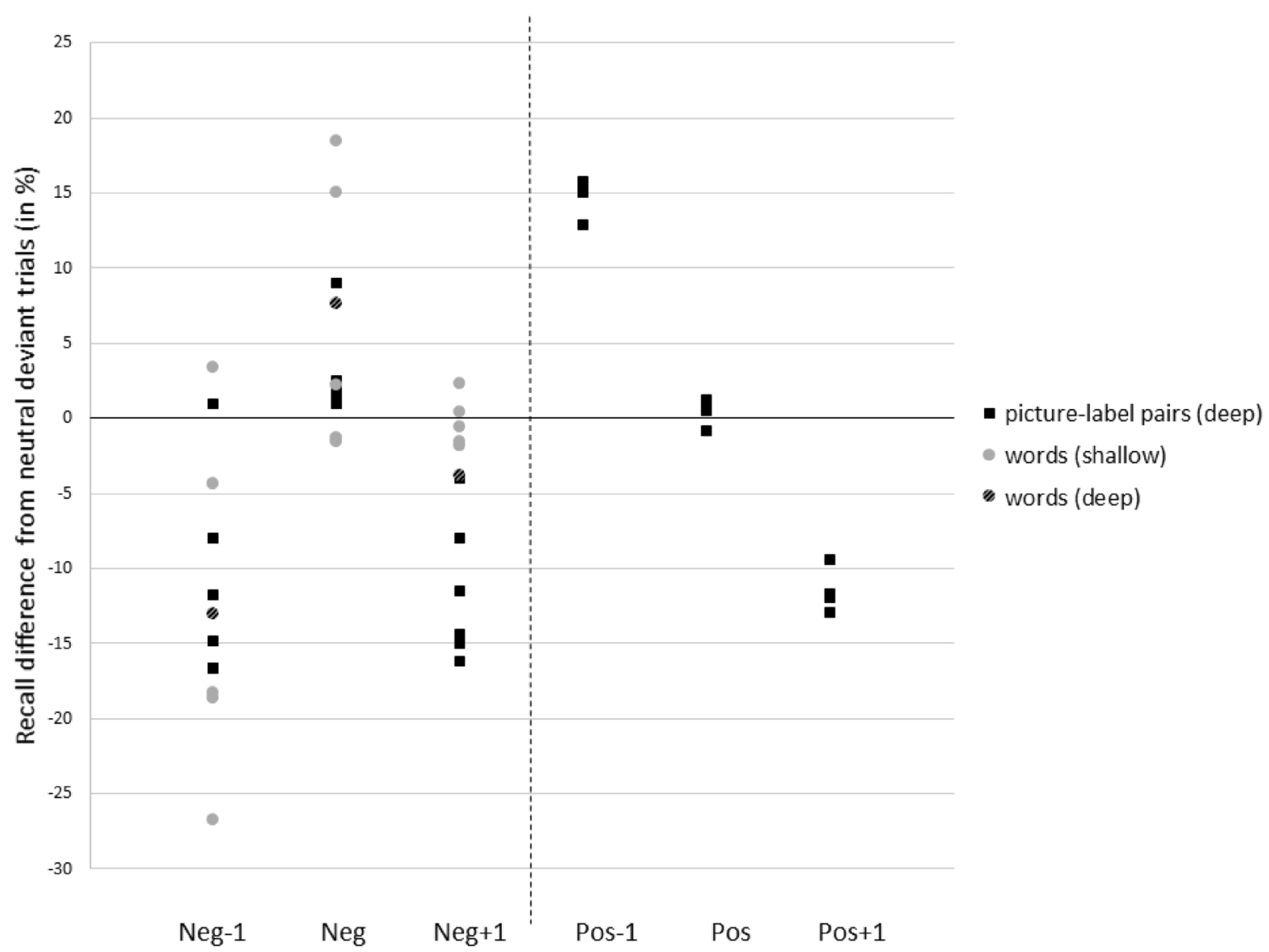

Fig. 3 Deviations of averaged recall rates for all investigated stimulus positions as compared with the averaged recall rates for the neutral deviant (in percent) from the respective EOP experiment (i.e., "contrastive method"). Included are only EOP experiments that involve an immediate free-recall task. Data for picture-label pairs are derived from Hurlemann et al. (2005: Exp. 1 and Exp. 2's placebo group; 2007a: healthy controls; 2007b: healthy controls) and Knight and Mather (2009), data for words are derived from Strange et al. (2003: Exp. 1, split in shallow and deep encoding, Exp. 2 and Exp. 3) and Strange et al. (2008: split for genotypes investigated). While all experiments using picture-label pairs employed deep encoding tasks (black quadratic figures), light gray round figures indicate that the word EOP used a shallow encoding task and the dark gray round figures stand for an experiment with words and a deep encoding task. Please note that exact values were available for all EOP experiments with pictorial stimuli, but nor for experiments with verbal material. In these cases, values had to be estimated from diagrams. Data from Miu et al. (2005) are not included because no differentiation was made between positive and negative deviants.

Positive values indicate hypermnesic effects (i.e., an enhanced recall rate for the respective position compared with the same position in the neutral deviant condition of the respective experiment), negative values indicate amnesic effects (i.e., a decreased recall rate)

Overview The presentation of arousing deviants in a sequence of non-arousing standard stimuli tends to influence the memory rate for stimuli neighboring the deviants (retrograde and anterograde memory effects) in a subsequent memory test. While both emotional deviant types - negative and positive deviants - tend to impair memory for subsequent stimuli (i.e., anterograde amnesia), the influence of emotional deviants on the memory performance for stimuli preceding a deviant depends on the valence of the deviant stimulus. Generally, negative deviants lower memory for stimuli presented before a deviant (i.e., retrograde amnesia), and positive deviants enhance memory for stimuli preceding a deviant (i.e., retrograde hypermnesia). Methods and results of the respective experiments are detailed below.

Detailed information Strange, Hurlemann, and Dolan (2003) investigated the effects of emotional stimuli on the recall performance of emotionally neutral standard stimuli. In a first experiment, subjects were required to memorize eight lists of 19 words. In each list, there was one perceptual deviant (written in another font than standard words), one semantic (not discussed further, as the results for this type of deviant were not reported), and one emotionally negative deviant word between semantically related standards. Participants were presented with words sequentially. Each trial began with the encoding of a list followed by an immediate free-recall task, in which they had to name as many words of the previous list as they could. Recall rates for deviant stimuli were significantly higher than for standard stimuli. However, perceptual and negative deviants had differential effects on memory for neighboring stimuli. While memory for neighboring standard stimuli was unaffected by perceptual deviants, negative deviants lead to retrograde amnesia (i.e., standard stimuli preceding negative deviants were remembered worse than other standard stimuli). This emotion-induced retrograde amnesic 


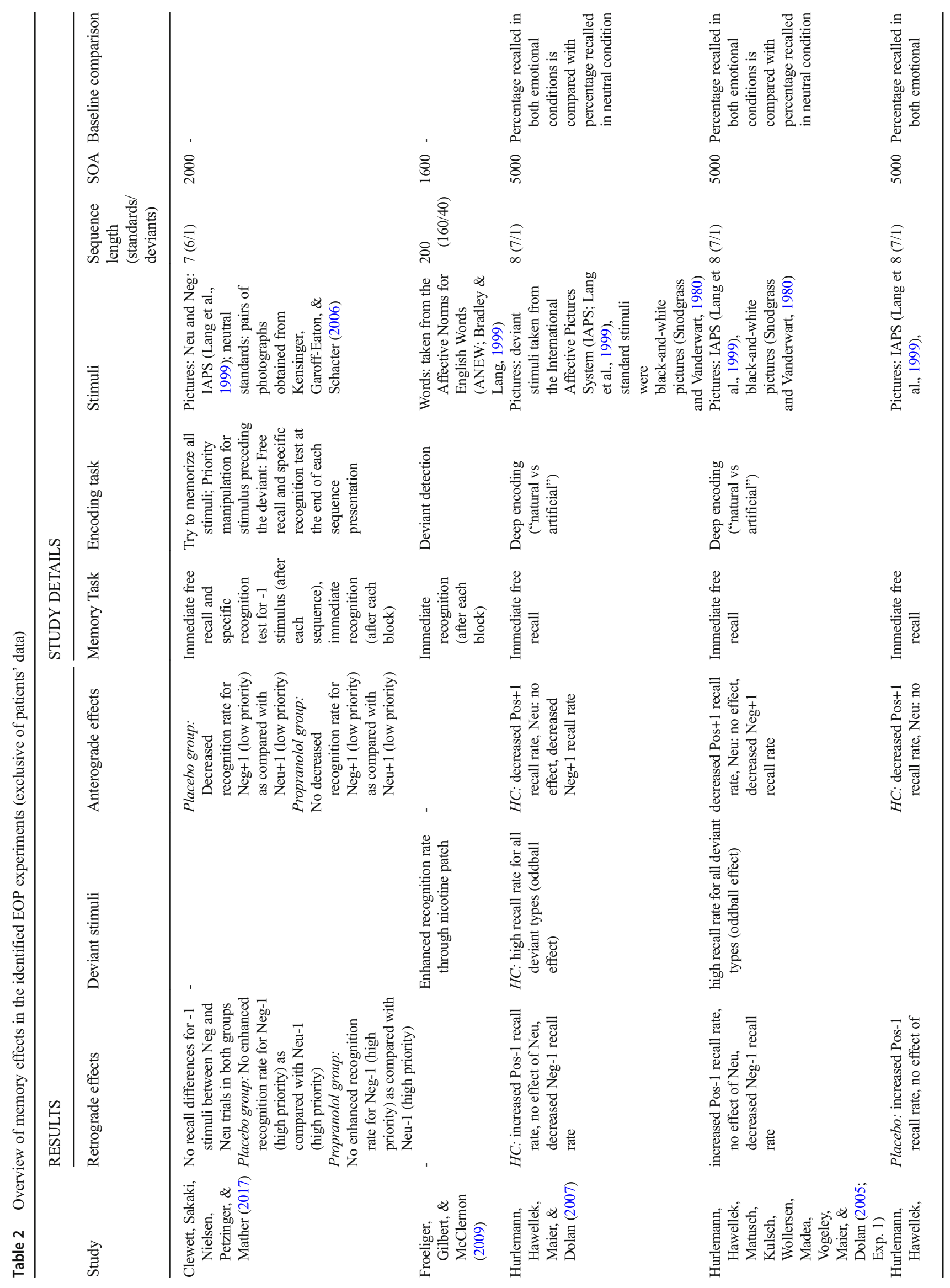




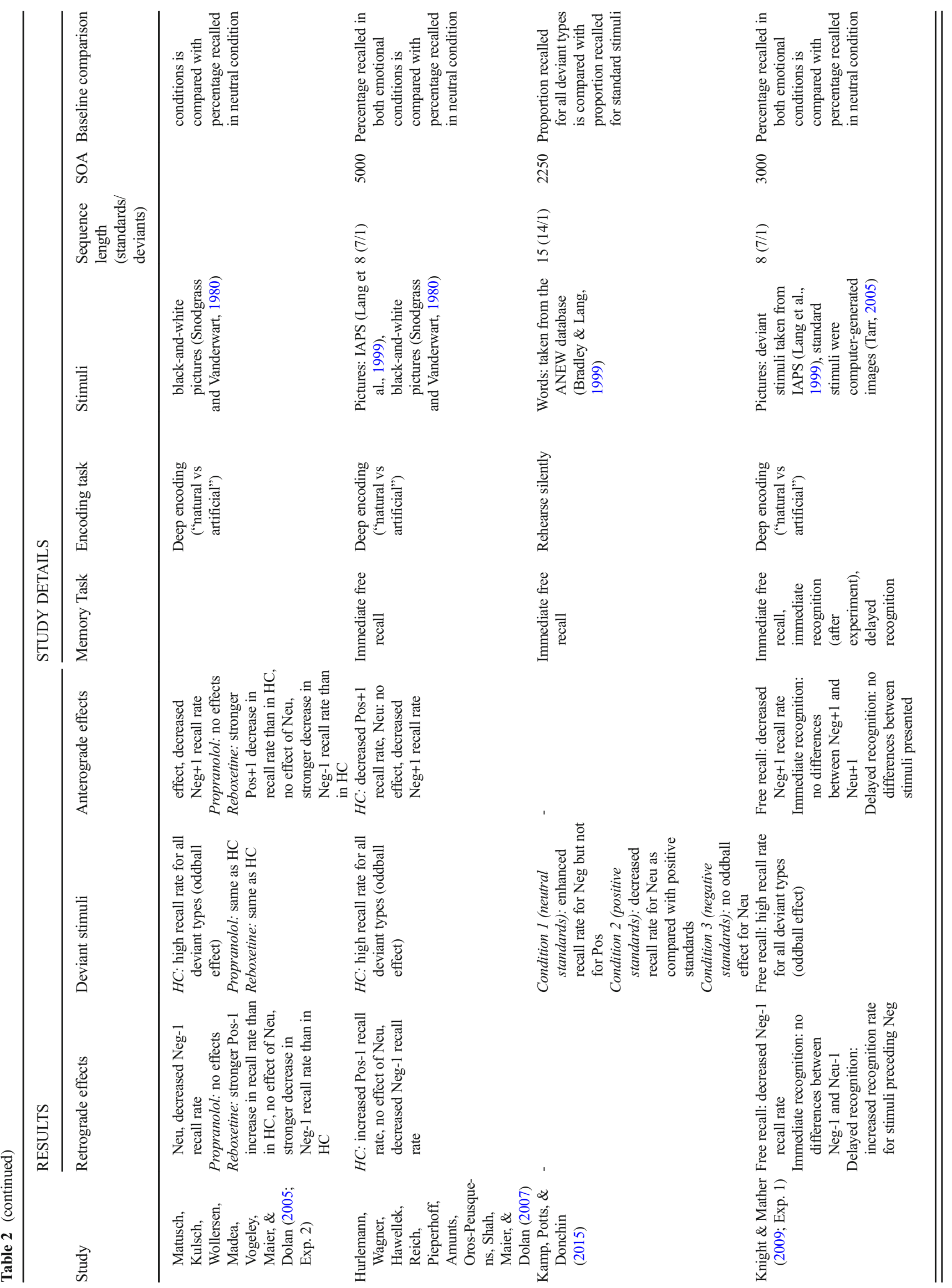




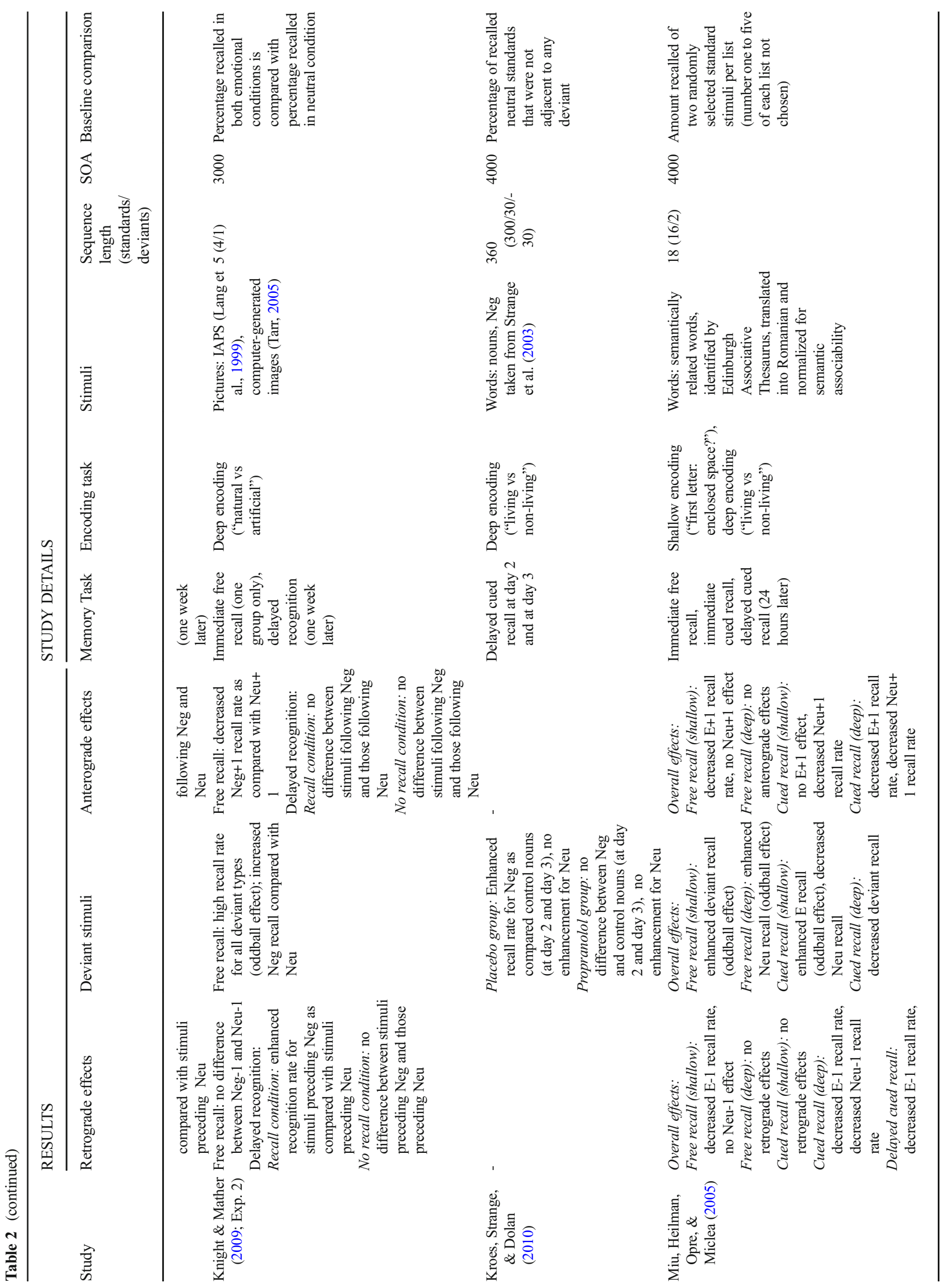




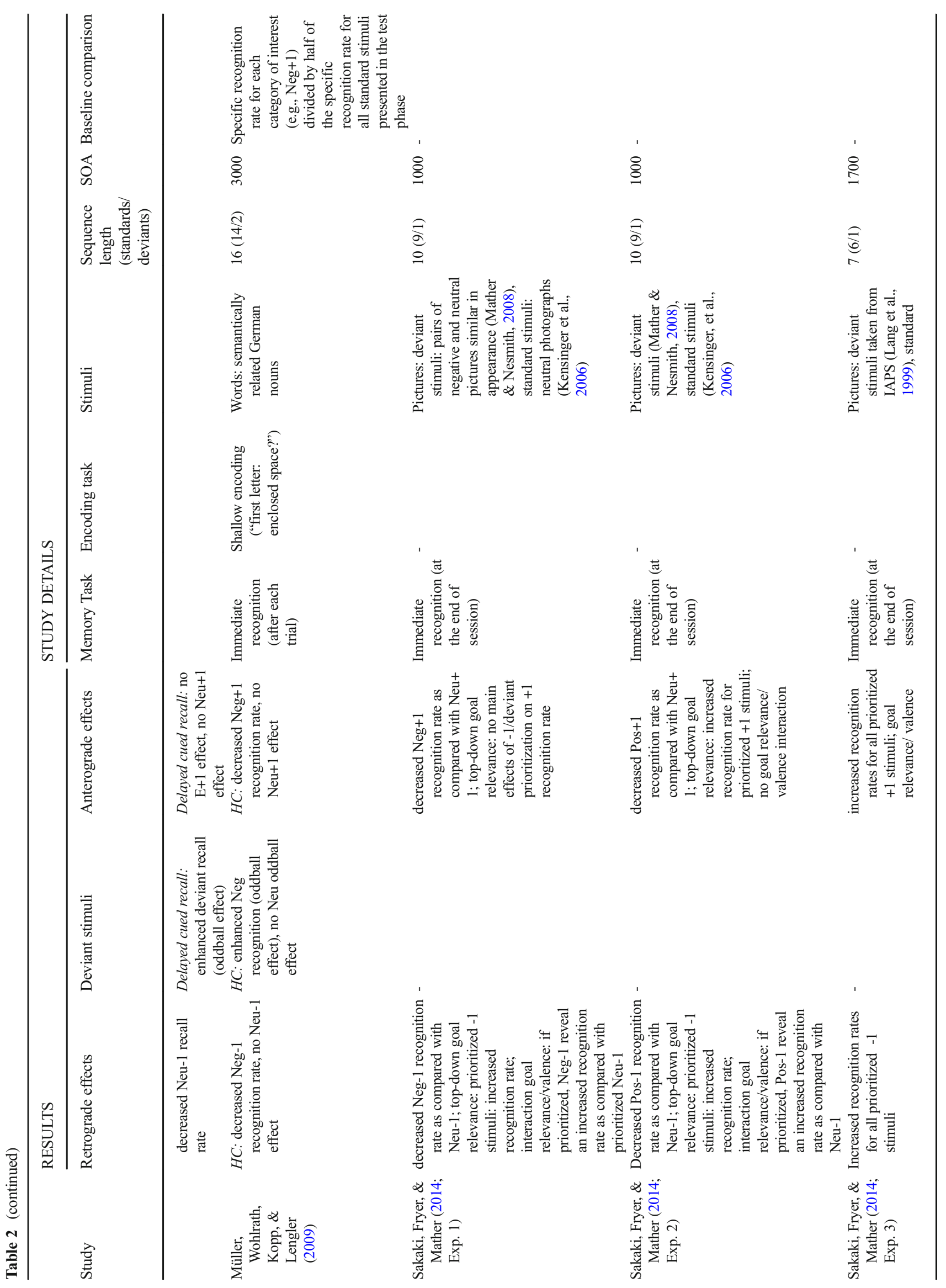




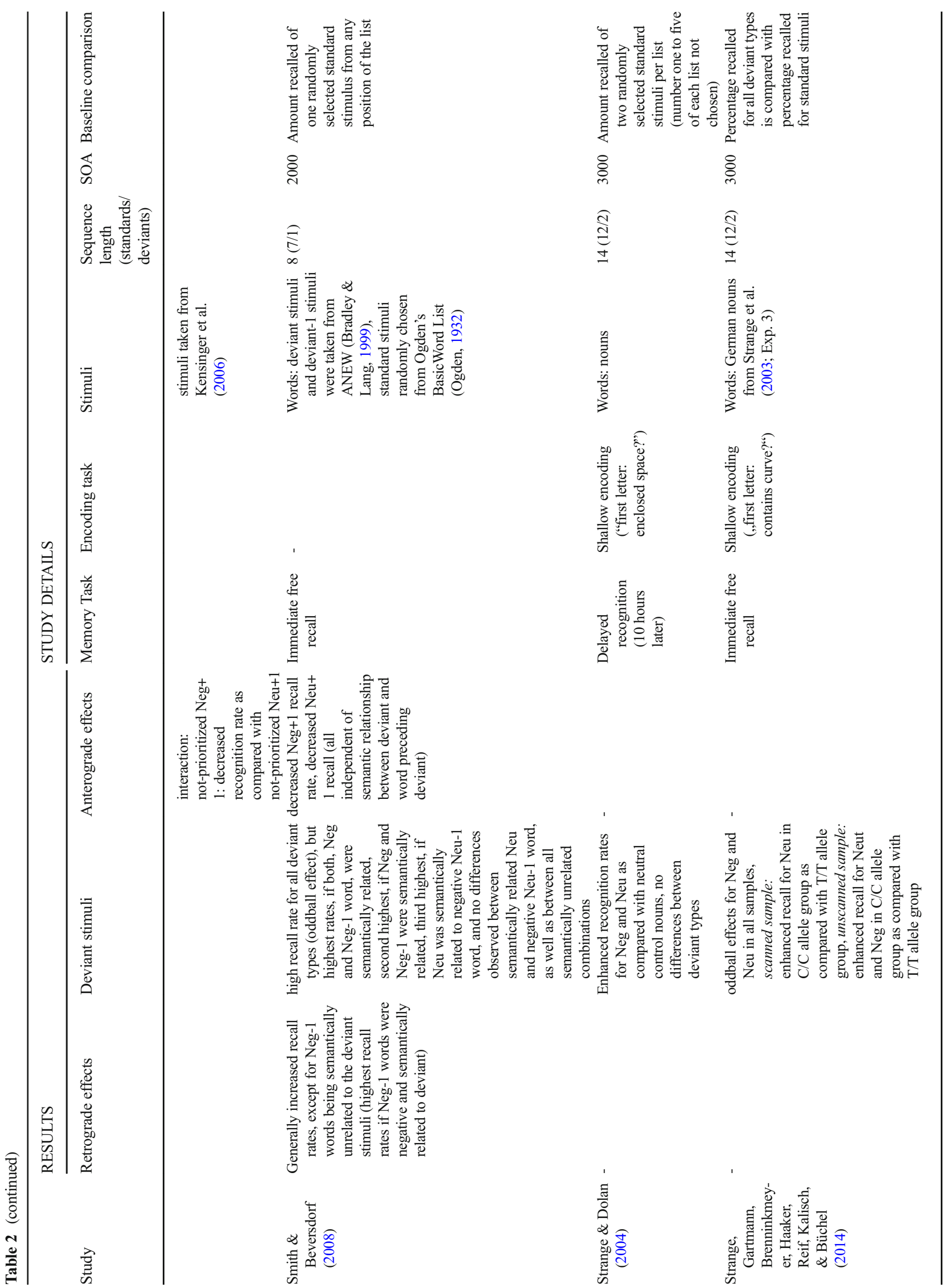




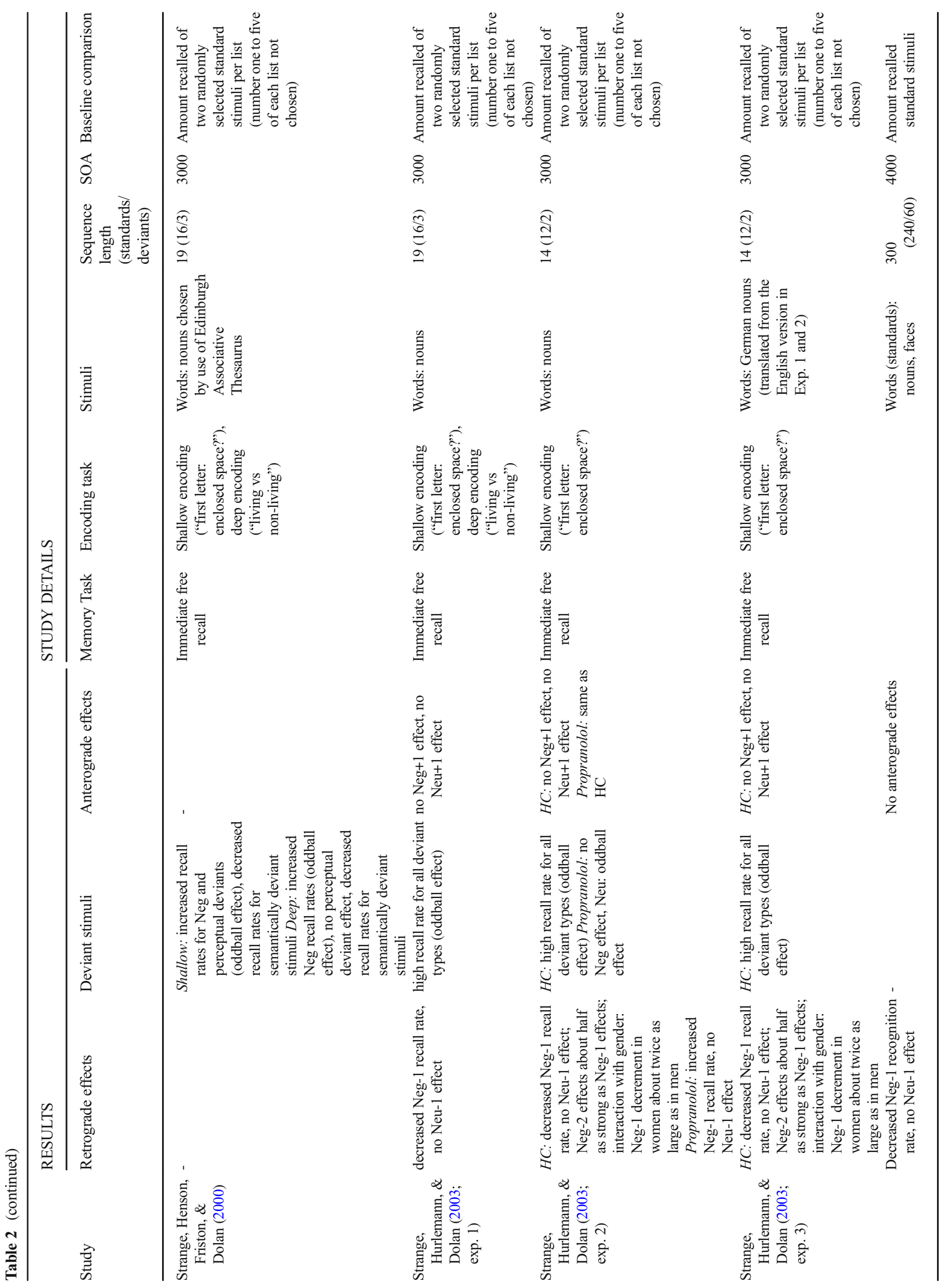




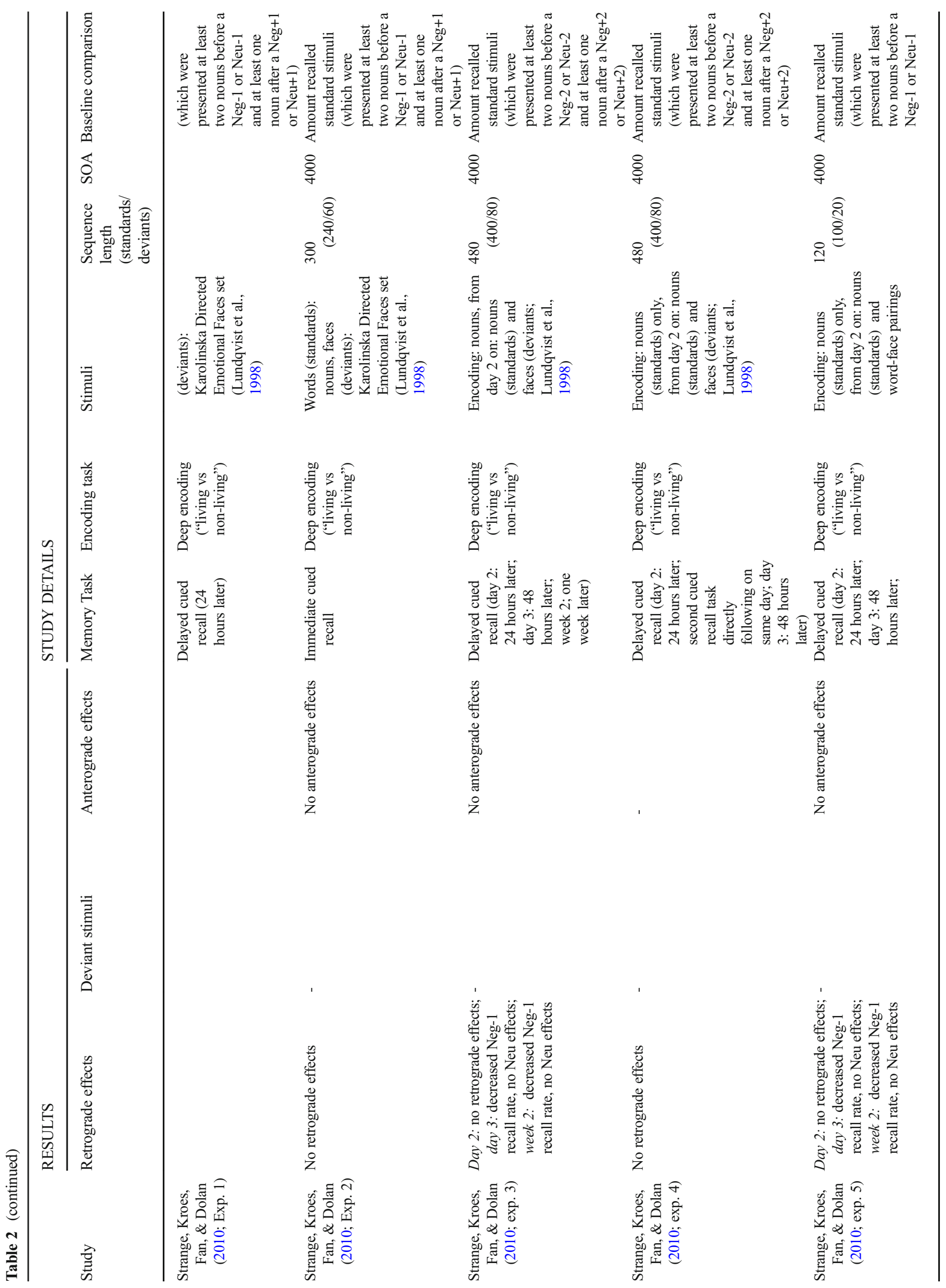




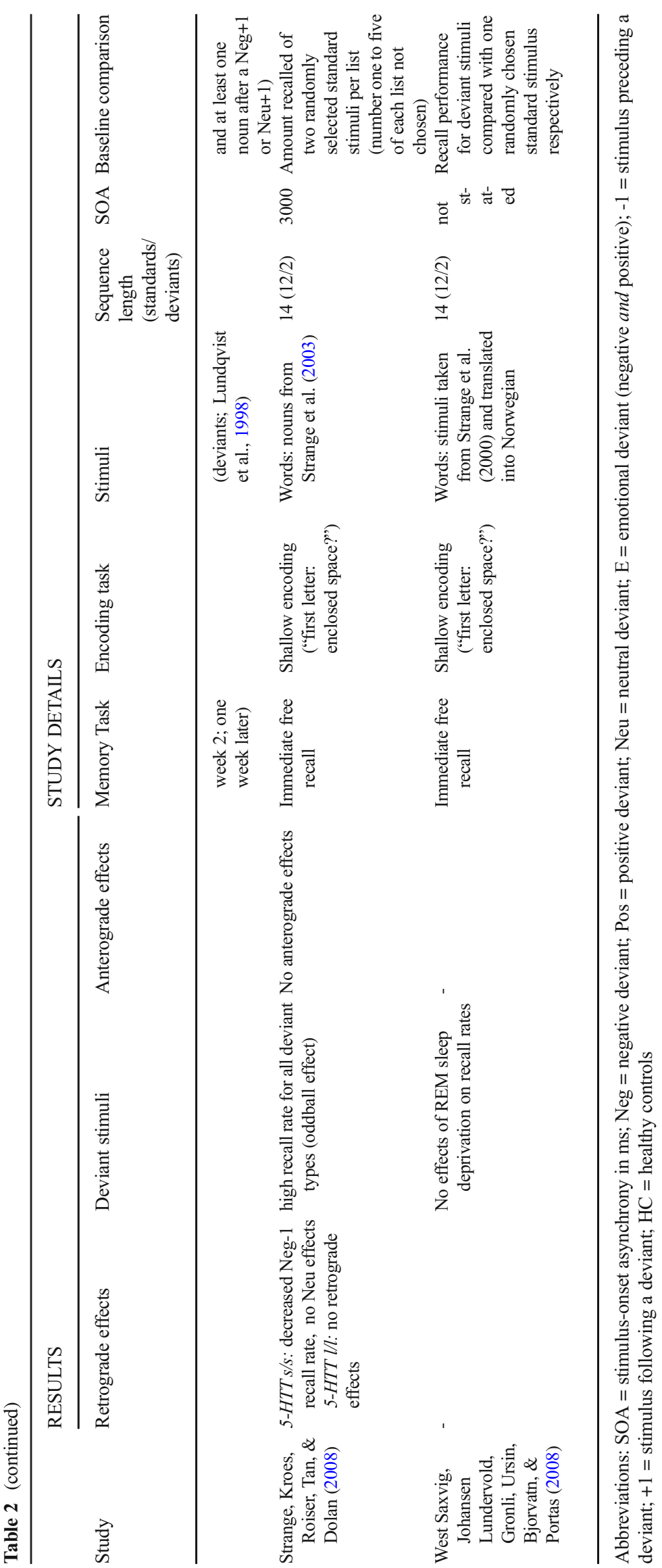


effect, which has been replicated using similar procedures (Miu et al., 2005; Strange et al., 2008) and with immediate recognition tests (Müller et al., 2009; in healthy controls), was not qualified by the type of encoding task (i.e. shallow vs. deep).

Hurlemann et al. (2005) used picture-label pairs and presented subjects with 36 lists, each containing seven black and white sketches as neutral standards and a single negative, neutral, or positive International Affective Picture System (IAPS) picture (Lang, Bradley, \& Cuthbert, 1999) as a deviant. After each list, a 30 -s distractor task was followed by a free-recall test. The emotion-induced retrograde amnesic effect was replicated.

Strikingly, analysis of the rate of recalled standard stimuli preceding emotionally positive deviant stimuli revealed a quite different pattern whereby memory for standard stimuli preceding positive deviants was enhanced compared with standards preceding neutral deviants (i.e., a retrograde hypermnesic effect). The retrograde amnesic effects of negative emotion and retrograde hypermnesic effects of positive deviants were replicated in two further experiments (in the healthy control groups; Hurlemann et al., 2007a, 2007b).

Additional analyses from Hurlemann et al. (2005, 2007a, 2007b) revealed anterograde amnesic effects. Standards following emotional deviants (both negative and positive) were recalled worse than standards following neutral deviants.

Hence, the results of these three studies (Hurlemann et al., $2005,2007 a, 2007 b)$ indicate a retrograde influence by emotion, which is valence-dependent. Note that the enhancing effects of positive deviants cannot be explained by differences in arousal since the positive deviant stimuli of all three studies were chosen to be equally arousing compared with negative deviants.

Using a slightly different design than in the experiments above, Strange et al. (2010) showed the influence of emotional deviants that are not part of the to-be-remembered stimulus material on the memory performance for sequentially presented neutral words at different stages (encoding: Exps. 1 and 2; retrieval/reactivation: Exps. 3, 4, and 5). In a series of five reported experiments, the authors presented their participants with emotionally neutral nouns sequentially. Noteworthy, all stimuli of the pool of 400 words were "stem-unique" in that the first three letters were unique for each noun. In Experiment 1, on day one, subjects were shown 240 of these nouns. Critically, 60 faces with either fearful or neutral facial expressions were interspersed as deviant stimuli after every third, fourth, or fifth noun. Participants' task was to classify each of the nouns as either "living" or "non-living" (deep encoding task) and to detect all the deviants (i.e., the faces) by buttonpress. Twenty-four hours later, participants engaged in a wordstem completion task (i.e., a cued recall task). A clear negative-emotion-induced retrograde amnesic effect was found, whereby recall was worse for words preceding fearful faces than words preceding neutral faces. Recall for words following a negative deviant was numerically but not significantly decreased. By contrast, neutral facial expressions did not evoke any differences in the recall rates of the words preceding and following the deviants as compared with control nouns. This result replicates the findings of retrograde (negative-)emotion-induced amnesia in the experiments reported above.

In Experiment 2, Strange et al. (2010) demonstrated that the negative-emotion-induced retrograde amnesic effect vanished if the cued recall task was completed immediately after the encoding phase. The absence of a retrograde effect induced by negative deviants seems to be in contrast to an earlier study by Strange et al. (2003), in which retrograde amnesia was found in an immediate free-recall task. The authors argue that this difference might have been due to the semantic relatedness between the verbal material in the 2003 experiments, and that those immediate effects on free recall might stem from "disruptions of category-cued retrieval strategies" (Strange et al., 2010; p. 3). However, while semantic links in the experiments from 2010 are clearly weak, it may be doubted that the words in the experiments from 2003 (Strange et al., 2003) really shared strong semantic links (see also Smith \& Beversdorf, 2008).

Although the difference in semantic relatedness between the experiments (Strange et al., 2003, 2010) can be questioned, other differences are easier to identify. It seems that the main differences were the presentations of faces as negative deviants instead of words and that a cued recall task was employed instead of a free-recall task as in the earlier experiments. While the actual reason for the absence of the amnesic effect is subject to speculation, it seems plausible that the cues (i.e., unique word stems) in the immediate cued-recall tasks simply were so strong that retrograde amnesic effects could not come into play in Experiment 2 (Strange et al., 2010). Like in Experiment 1, there was a numerical but not significant anterograde amnesic effect.

In Experiment 3, participants engaged in the encoding session, which was performed three times to enhance retrieval success in the following recall tasks. Contrary to the first two experiments, no faces were presented in the encoding session of the third experiment. On day two, participants completed the normal word stem completion task. This time, however, the subjects also had to detect (fearful and neutral) faces that were interspersed as deviant stimuli during recall. Thus, beside the word stem completion, participants' second task was deviant detection. On day three, as well as 1 week after the first experimental session, participants again completed the word stem completion task (these times without deviant stimuli). Results of the word stem completion task on day two revealed no differences regarding recall rates between stimuli preceding and following faces during recall and the recall rates for control nouns. Interestingly, on day three as well as 1 week 
later, participants completed fewer word stems of words remembered on day two that had preceded fearful faces in the cued recall session of day two (compared to control nouns; i.e., retrograde amnesic effect). No such effects were found for words preceding neutral facial expressions. This basic pattern of findings was replicated using a slightly different method in Experiment 5.

To rule out the possibility that this effect was due to impaired novel encoding of reactivated memories, Experiment 4 was designed, with two word-stem completion tasks on day two (the first with intermingled faces, the second without the presentation of faces). The last word-stem completion session took place on day three. Again, emotion-induced retrograde amnesia occurred in the word-stem completion task on day three (i.e., only for words which were presented before intermingled fearful faces), and there were no differences in the second word-stem completion task of day two between words previously (i.e., in the first word stem completion task of day two) preceding fearful and neutral faces.

Taken together, the findings by Strange et al. (2010) provide evidence for an effect of emotion on the reconsolidation of memories after successful reactivation. The authors suggest that the retrograde amnesia in delayed cued-recall tasks is due to a disrupted integration of retrieved nouns by the presentation of fearful faces. Importantly, this effect cannot be obtained by the presentation of neutral faces and is time-dependent (Exp. 2) with the effect not occurring if the recall task with interspersed faces was conducted on the same day as the cued recall task without faces.

In summary, the evidence reviewed in this section indicates that arousing deviant stimuli in a sequence of non-arousing neutral standards affect the memory rate for neutral stimuli neighboring the deviants in immediate free-recall tasks and immediate recognition tasks. The analysis of only one experiment, in which an immediate cued recall task was employed (Strange et al., 2010, Exp. 2), did not reveal emotion-induced effects. Thus, the emotion-induced memory effect on neighboring information might be one further example of an effect that is task-dependent (Roediger III, 2008).

It seems, however, that the deviants' emotional valence may play a crucial role for the influence they have on neutral stimuli directly preceding the deviants: While there is a large body of evidence showing that negative deviants lead to retrograde amnesic effects, there are three studies, in which positive deviants are presented, indicating that positive deviants lead to retrograde hypermnesic effects. Hurlemann et al. (2005) speculated that this retrograde enhancement is caused by a psychological mechanism that "promotes retention of information that predicts reward" (p. 6348). Following this logic, stimuli that served as cues for negative events in the past should be related to forgetting instead of remembering the particular instance and its cues. However, one could argue that forgetting the cues that have put an organism at risk is rather not adaptive. Nonetheless, the findings by Hurlemann et al. (2005) highlight the importance of valence, which apparently can act as a moderator variable in EOP studies on memory.

With respect to anterograde effects, the results reviewed in this section imply a general tendency towards an anterograde amnesic effect of emotional (negative and positive) deviants that is absent if emotionally neutral deviants are presented instead.

The influence of semantic relatedness Another important variable - besides emotional arousal/valence - that could potentially modulate memory effects in the EOP is semantic relatedness (i.e., the ease with which an association can be established between two or more of the presented stimuli of a sequence). While in most EOP experiments the semantic relationship was held constant throughout the sequence (meaning that either all stimuli were unrelated or related), one experiment manipulated the semantic relationship between the standard stimulus preceding the deviant and the deviant (Smith \& Beversdorf, 2008). The results underline the importance of semantic relatedness in memory experiments and have interesting methodological and theoretical implications.

Overview If standard stimuli presented directly before a deviant are semantically related to this deviant stimulus, memory rate for these standard stimuli is enhanced (i.e., retrograde hypermnesia). This effect was found for both negative and neutral deviant trials.

Detailed information Smith and Beversdorf (2008) presented subjects with 48 lists, each comprised of seven standards, written in white on a black background, and a single deviant, written in lime green. Six of the standards were always emotionally neutral and semantically unrelated to each other. The deviant and the standard immediately preceding the deviant could each be either neutral or negative and they could be either semantically unrelated or related to each other (they were always unrelated to the other standards). The SOA of all stimuli was 2,000 ms. Each list of the encoding stage was immediately followed by a free-recall task. The mean recall for each position of interest (stimuli directly preceding the deviant, the deviant itself, and stimuli directly following the deviant) was compared with control words recall (recall of one randomly selected neutral standard of each list that was not presented at a position of interest; "compare with standard method," see above).

Summing up the results, we focus on four of these eight conditions, in which the stimuli preceding the - either negative or neutral - deviants are neutral plus either related or unrelated to the deviants. The condition in which a semantically related neutral standard preceded a negative deviant 
produced the highest deviant recall and a clear retrograde hypermnesic effect. The condition in which a semantically related neutral standard preceded a neutral deviant also revealed a retrograde hypermnesia and a clear oddball effect. However, the oddball effect for the neutral deviants was about half as big as in the condition in which negative deviants were presented. In conditions that both included only unrelated stimuli, the oddball effects were equally strong and did not differ from the oddball effect in the negative deviant condition with a related pre-deviant stimulus. By contrast, in both of these conditions the retrograde hypermnesic effect vanished and recall rates did not differ significantly from control words recall. Further, irrespective of the emotion of the deviant or the semantic relatedness of the word preceding the deviant, all stimuli directly presented after a deviant stimulus were recalled significantly worse than control words (i.e., an anterograde amnesic effect).

This experiment (Smith \& Beversdorf, 2008) highlights the importance of semantic relatedness. It is shown that if neutral standards that precede a deviant are - unlike the other neutral standards presented in a sequence - semantically related to the deviant, the recall rate is enhanced compared with the recall for neutral standards preceding a semantically unrelated deviant. This advantage of related words occurs in both sequences with negative deviants and sequences with neutral deviants. Possible reasons for the better recall of related words are an encoding advantage (activation of semantic memory might result in a more efficient encoding of the semantically related pair of words) as well as a recall advantage (the recall of one of the related words might serve as a cue for the other word).

At first glance, the results for unrelated words seem to be at odds with the typically observed retrograde amnesic effects in experiments with written verbal material (e.g., Müller et al., 2009; Strange et al., 2003). However, the absence of retrograde amnesic effects might be a result of the relatively low number of words within a sequence. While in other EOP studies with words 18 or more words per list were presented, Smith and Beversdorf (2008) used only eight words per sequence. Thus, the retrograde impairment might not have been strong enough and was presumably countered by rehearsal strategies of the participants.

Taken together, the results of this section highlight the importance of a careful choice of the to-be-presented stimuli within a sequence, since semantic links between a neutral standard and a deviant can lead to a strong modulation of the memory rate of a neutral standard stimulus that can potentially undermine the actual emotion-induced effects. Second, the experiment shows that semantically linking a neutral stimulus with a deviant causes a memory benefit for the neutral stimulus. This finding could be explained by a salience enhancement through the connection with a high priority stimulus (i.e., the deviant). We address the related issues of prioritization in the following section.
The influence of "attentional weights" and goal relevance The third factor highlighted in this review is goal relevance. It plays an important role in experiments in which participants are told to prioritize certain stimuli of the sequence depending on their position (i.e., stimuli preceding or following the deviant, respectively). EOP experiments reported in this category emphasize the interplay of saliency and emotional arousal, as prioritized stimuli gain a memory advantage, but only if arousal induction took place by the presentation of an emotional rather than a non-emotional deviant. Like in the case of semantic relatedness, evidence for the influence of goal relevance has important implications both on an experimental and on a theoretical level, and is discussed in the subsequent sections.

Overview The likelihood of remembering stimuli surrounding a deviant depends on the saliency of these standards. If, for example, a neutral stimulus preceding a negative deviant was made more salient by task instruction, the memory rate for this standard stimulus would rise, and not decline as one would usually expect (see the subsection on "The influence of emotional valence/arousal" above). This can be explained by arousal-biased competition (ABC) theory (Mather \& Sutherland, 2011; see also "Theoretical background of amnesic/hypermnesic effects of emotional deviant stimuli" below).

Detailed information So far, a large body of evidence has been reviewed indicating that the presentation of negative deviants leads to weaker memory for preceding neutral standards (retrograde amnesia). However, using an experimental design similar to the EOP, Anderson, Wais, and Gabrieli (2006) found enhanced memory for neutral stimuli directly preceding negative stimuli (hypermnesic effect). Knight and Mather (2009) conducted two experiments with the aim of determining when negative stimuli lead to retrograde amnesic or hypermnesic effects. Experiment 1 used a design similar to Hurlemann et al. (2005) with the main exceptions being that Knight and Mather (2009) only used emotionally neutral (perceptual) and negative deviant stimuli but not positive deviants, and that their participants took part in an old/new recognition task either immediately after the experiment or 1 week after the first session, in addition to immediate free recall after each list. In the recognition test, subjects were presented with two pictures from a single category including their captions (e.g., two different guitars). They could either report that one of the pictures was old or that they were both new. When presented with an old category (e.g., two guitars and one of the guitars was on the original list) the researchers considered specific recognition to be an old response to the exact same picture and general recognition 
to be an old response to either the exact same picture or the foil picture, as long as it came from an old category ${ }^{1}$.

Results showed negative-emotion-induced retrograde and anterograde amnesic effects for free recall, and specific recognition in the immediate and delayed recognition tests. However, negative-emotion-dependent decrements in the recognition tests were limited to specific recognition (i.e., there was no difference in retrograde and anterograde general recognition rates between the negative and the neutral deviant condition). Knight and Mather (2009) also analyzed recognition rates separately for stimuli that had been recalled in the immediate recall test and stimuli that had not been recalled. Strikingly, after a 1-week delay, a negative-emotion-induced retrograde hypermnesia (in comparison with standard stimuli preceding neutral deviants) was apparent for items that had been recalled 1 week before. This effect did not hold true for stimuli that had not been recalled in those recall tests. Knight and Mather (2009) explain this by a "mnemonic boost" that is received by stimuli because of their relative attentional weight during the induction of arousal. Stimuli that were recalled against the tendency of a decrease of recall for stimuli preceding emotional oddballs - seemingly possessed a high attentional weight compared to those that were not recalled. Those stimuli surviving the retrograde amnesic effect then profited from enhanced consolidation and were more likely to be recognized after a 1-week delay.

In Experiment 2, Knight and Mather (2009) directly manipulated the items' representations by requiring one half of the participants to immediately recall the words of a list at the end of each trial (high attentional weights for all stimuli of the sequence), while the other half was not assigned to this freerecall condition (low attentional weights for all standard stimuli). After a 1-week delay, all participants took part in a recognition test, which confirmed retrograde emotion-induced enhancements through negative deviants but only for the group of participants, which previously had had to recall the items in the first session. For the group of participants, which did not have to recall items in the first session, there was no difference between the recognition of neutral standards preceding neutral deviants and neutral standards preceding negative deviants.

Sakaki, Fryer, and Mather (2014) investigated whether topdown goal relevance could be one determinant of whether retrograde amnesia or enhancement would occur. In their Experiment 1, the authors used emotionally negative as well

\footnotetext{
${ }^{1}$ Note that this division in "general" and "specific" recognition makes sense if one refers to the stimulus as a whole (including the picture and its caption). However, it may be criticized for the fact that in order for a participant to make a correct old/new decision it is not even necessary to look at the pictures at all. Thus, a correct "old" judgment on a similar but not the exact same picture presented in the learn phase might not necessarily reflect general recognition of the whole stimulus, but rather specific recognition of the word written underneath the picture.
}

as neutral picture-label pairs with a thick black frame as deviant stimuli and neutral picture-label pairs without frames as standards. During the learn phase, subjects were instructed to pay special attention to either the picture in the black frame or the picture appearing before the picture with the black frame. After each list subjects had to recall the name of the picture that they were supposed to focus on. After the presentation of ten lists of semantically unrelated stimuli, participants completed an old/new recognition task.

The impact of deviant stimuli on the recognition performances depended on the participants' focus: If they had been instructed, prior to encoding, that they were to especially focus on the deviant stimuli, emotion-induced retrograde amnesia occurred. By contrast, if participants had been asked to focus on the pictures appearing directly before a deviant stimulus, the presentation of negative deviants led to retrograde hypermnesia. In addition, negative deviants but not neutral deviants had an anterograde amnesic influence on the recognition of succeeding standards, irrespective of prioritization condition.

In Experiment 2, Sakaki et al. (2014) showed that retrograde (amnesic/hypermnesic) effects of positive deviant stimuli were modulated by prioritization/focus, which mirrored the results for negative deviant stimuli. They found a similar pattern of results when positive deviants were used, whereby memory for standard stimuli preceding positive deviants was enhanced (retrograde hypermnesic effect) when they were prioritized during encoding, but was impaired when deviants were prioritized during encoding (retrograde amnesic effect). In contrast to Experiment 1, however, Sakaki and colleagues had included a condition in which participants were to focus on stimuli following the deviants. As expected, there were anterograde amnesic effects of positive deviants (as compared with neutral deviants) on following standard stimuli, if participants did not have to focus on the succeeding standards. However, against the authors' prediction, there was no anterograde hypermnesia in the focus-on-subsequent-stimulus condition for positive deviant trials as compared with neutral deviant trials.

In Experiment 3, the differential influences of valence and arousal were highlighted. Similar to the previous experiments of their study, Sakaki et al. (2014) presented participants with lists of eight picture-label pairs. One of the pictures was either a negative, positive, or neutral deviant picture presented at position four, five, or six. In the encoding phase, every list was followed by a cue to retrieve the prioritized item (either the stimulus before or after the black-framed picture-label pair, which was varied between subjects). After all lists had been presented, participants completed a distractor task (counting back from a three-digit number in steps of three for $1 \mathrm{~min}$ ), and then performed an old/new recognition task. 
Again, retrograde hypermnesic effects were shown if predeviant stimuli were to be prioritized but retrograde amnesia occurred in the focus-on-stimuli-succeeding-the-deviants group. However, Sakaki et al. (2014) did not find a priorityinduced hypermnesia for to-be-prioritized standard stimuli appearing directly after both a negative and a positive deviant as compared with standards succeeding a neutral deviant. By contrast, in cases when the succeeding standard stimuli was not focused on, the recognition rate for those standards was decreased if the preceding deviant was either negative or positive as compared with neutral deviant trials (i.e., anterograde amnesia). At the end of Experiment 3, participants provided arousal and valence ratings of each deviant stimulus. This enabled the authors to analyze the impact of arousal and valence in a hierarchical linear model (HLM) with each trial as a level-1 unit and each participant as a level-2 unit. An arousalby-priority interaction reached significance, indicating that arousal (but not valence) influences the impact of priority on the memory performance of neutral standards preceding negative and positive deviants.

One further investigation of priority effects (Clewett et al., 2017) included an EOP with picture-label pairs. In this experiment, participants viewed sequences of seven semantically unrelated pictures. One deviant per sequence appeared at the third, fourth, or fifth position and was always presented on a black screen to be perceptually different from emotionally neutral standards that were presented on a white background. Additionally, deviants were either emotionally neutral or negative (IAPS pictures; Lang et al., 1999). Participants were instructed to remember as many neutral stimuli as possible and to especially focus on the stimuli preceding the black-framed deviants (i.e., to prioritize neutral standards presented directly before the deviants). After each sequence, participants were prompted to report the label of the to-be-prioritized stimulus, and then to answer a forced-choice question regarding a perceptual detail of this stimulus (free-recall task). At the end of each block that consisted of six sequences (including three negative and three neutral deviant trials), recognition memory was tested by an old/new recognition task which involved testing recognition performance for neutral standards preceding and following deviants.

This experiment included two groups of participants, one placebo group and a group of participants who were given propranolol (a drug that is known to influence amygdala activity; for a description of results of this group see the following section). Results in the placebo group showed no difference between trials with negative and with neutral deviants regarding free recall or (specific) recognition accuracy for stimuli preceding the deviants. However, there was a significant negative-emotion-induced anterograde amnesia. Thus, contrary to the results reported by Sakaki et al. (2014), emotional arousal induced by the presentation of negative deviants did not lead to a hypermnesia of prioritized neutral information that appeared directly before the deviant stimulus.

The evidence summed up in this section suggests that the occurrence of amnesic and/or hypermnesic effects in the EOP does not only depend on emotional valence and/or arousal of the deviants used. Instead, the influence of a presented emotional deviant on the memory performance of neighboring standard information changes depending on what is the participants' focus or goal. Knight and Mather (2009) were able to show that needing to remember a neutral standard neighboring a deviant in an immediate recall task can enhance its probability of recognition in a delayed recognition task. However, this benefit was only observed if the given standard information had been presented in a sequence including a negative rather than an emotionally neutral deviant. The authors explained this difference by an elevated attentional weight for standards in the recall condition, through the participants' attempt to rehearse the items during presentation. Stimuli presented before a deviant could especially profit from the attempt to rehearse because they (a) either had the highest attentional weights at the moment when emotion-induced arousal came into play or (b) became a marker of a negative event, and thus more significant/salient as compared with other items (note that this idea is similar to that proposed by Hurlemann et al., 2005, used to explain why there was enhanced memory for neutral standards preceding positive deviants). In a similar vein, Sakaki et al. (2014) argue that the induction of emotional arousal (regardless of valence) leads to enhanced memory for prioritized stimuli preceding a deviant but to decreased memory for not-prioritized stimuli preceding a deviant. However, Clewett et al. (2017) failed to replicate this pattern of results. With respect to anterograde effects, it seems that neutral standards following any deviant (this includes perceptual and emotional deviants) tend to be remembered less well, irrespective of the participants' focus.

\section{Physiological and pharmacological evidence}

Overview EOP experiments using drug administration in order to decrease or to amplify norepinephrine (NE) release in the human brain as well as experiments with patients suffering from amygdala lesions show that emotion-induced memory effects may be dependent on $\beta$-adrenergic connections and a normally functioning amygdala.

Detailed information The typical finding of negative-emotioninduced retrograde amnesia was shown to be modulated by the administration of a psychoactive drug, namely propranolol hydrochloride (Strange et al., 2003; Exp. 2). The authors replicated their results of Experiment 1 (see above) in the placebo group and extended their findings by administering propranolol, a substance causing a $\beta$-adrenergic blockade, i.e., a blockade of central norepinephrine (NE) release (Cahill, 
Prins, Weber, \& McGaugh, 1994). NE that is released in the amygdala during the perception of emotional events plays an important role for the advantage of emotional over nonemotional stimuli in memory (Markovic, Anderson, \& Todd, 2014).

Results of Experiment 2 (Strange et al., 2003) indicated that the negative oddball effect present in the placebo group was absent in the propranolol group. In addition, the typically observed retrograde amnesia for neutral stimuli preceding negative deviants was not only absent but even reversed, thus resulting in a retrograde hypermnesic effect in the propranolol group. Note that propranolol only affected the recall performance for negative deviants and stimuli directly preceding negative deviants. Recall rates for stimuli following negative deviants as well as recall for perceptual deviants and their neighbors were unaffected by the administration of propranolol. Also, while a higher recall of negative deviants in the placebo group was coupled with a lower recall of stimuli preceding the negative deviants on an item-to-item basis, this coupling was absent in the propranolol group.

In Experiment 3, it was shown that a patient suffering from Urbach-Wiethe disease (a disorder accompanied by an amygdala lesion) exhibited neither the emotion-induced retrograde amnesic effect nor an enhanced recall rate for aversive oddball stimuli. The authors concluded that the reported emotioninduced retrograde amnesia is both amygdala- and $\beta$-adrenergic-dependent.

Further support for the importance of these factors stems from an fMRI study (Strange \& Dolan, 2004), in which it was shown that successful encoding of negative deviants in an EOP was correlated with amygdala activation during presentation. This did not hold true in participants who had been administered the $\beta$-adrenergic antagonist propranolol.

In another experiment involving drug administration (Hurlemann et al., 2005; Exp. 2), one-third of the participants were given propranolol hydrochloride (which blocks central NE release, see above), another third received reboxetine (a substance that enhances central NE release), and another group of participants were given a placebo. The only difference in the experimental design to Experiment 1 (see above) was that participants had to rate all deviant pictures used in the experiment on the dimensions valence and arousal after the experiment. Analysis of the placebo group revealed the same effects as in the first experiment, replicating both the valence mediated retrograde amnesic effects (if negative deviants had been presented) and retrograde hypermnesic effects (if positive deviants had been presented). However, the administration of propranolol abolished all emotion-induced retrograde and anterograde memory effects. By contrast, reboxetine enhanced the previously reported effects on the recall rate of standard stimuli, almost doubling the size of the retrograde and anterograde effects observed in the placebo group. Results of the valence and arousal ratings of the three types of deviant stimuli indicated that the administered drugs altered only the perception (thus rating) of arousal, but not that of valence: Participants in the propranolol group gave lower ratings for both negative and positive deviant stimulus types with respect to emotional arousal than those in the placebo group, while the arousal ratings of participants who had been given reboxetine were significantly higher for emotional deviants. The arousal ratings for emotionally neutral deviant stimuli and all valence ratings were unaffected by drug administration. These findings indicate that (high) arousal is a prerequisite of the emotion-induced retrograde and anterograde memory effects. In addition, it seems that arousability (and not the perception of valence) is modulated by NE release. With regard to the retrograde hypermnesic effects of positive deviants, Hurlemann et al. (2005) speculate that subdivisions of the prefrontal cortex might inhibit arousal-related disruptions of retrograde recall (Dolcos, LaBar, \& Cabeza, 2004; for a review see Hurlemann et al., 2008).

In an experiment also reported above, Clewett et al. (2017) investigated effects of prioritization on memory for standard stimuli surrounding (emotional) deviants. They administered propranolol to one-half of the participants in order to test whether $\beta$-adrenoreceptor activation can facilitate memory consolidation of goal-relevant, emotionally neutral standards. In the placebo group, anterograde amnesic effects occurred for the not-to-be-prioritized stimuli succeeding a negative deviant, while no enhancements or decrements were observed for the prioritized neutral standards preceding a negative deviant. In the propranolol group, however, analyses revealed neither anterograde nor retrograde memory effects. The authors discuss their results with respect to the "glutamate amplifies noradrenergic effects" (GANE) model (explained in detail in Mather, Clewett, Sakaki, \& Harley, 2016). However, this explanation is subject to current debate, with authors proposing an alternative model (Strange \& Galarza-Vallejo, 2016) that assumes non-linear shifts in locus coeruleus (LC) activity as the underlying mechanism for the bi-directional effects of negative deviants on preceding neutral standard information.

\section{Summary of evidence}

The results as well as the underlying mechanisms that potentially influenced the results may differ largely between experiments in which immediate memory tasks (i.e., tasks that are either to be done at the end of each trial, at the end of one experimental block or at the end of the experimental session) and those in which delayed-memory tasks are employed (i.e., tasks with a delay of days and even weeks). The reported results of immediate memory tasks allow certain generalizations, whereas results of delayed tasks are more specific for the individual authors' hypotheses. Here we sum up the results separately for immediate and delayed-memory tasks. 
Performance in immediate memory tasks Our investigation of the reported results concerning EOP memory experiments allows us to make four generalizations about the results of immediate memory tasks: (1) oddball effects, (2) retrograde amnesic effects on standards preceding negative deviants, (3) anterograde amnesic effects on standards succeeding emotional deviants, and (4) amygdala-/arousal-dependency of the effects.

The first generalization is that there is a consistent mnemonic benefit for deviant stimuli as compared to standards, reflecting the oddball/von Restorff effect (von Restorff, 1933; Wallace, 1965). There are generally no differences in recall/ recognition between emotional and emotionally neutral deviants (but note that in many experiments ceiling effects occurred, leaving no space for potential differences to develop; see also below: What makes an oddball odd?).

The second generalization is that, all else being equal, negative deviants impair memory for preceding neutral standards (e.g., Hurlemann et al., 2005; Knight \& Mather, 2009; Strange et al., 2003). This retrograde amnesic effect may, however, be reversed if the standard preceding the negative stimulus is made especially salient (by means of semantic relatedness of preceding stimulus and deviant: Smith \& Beversdorf, 2008; by means of prioritization of preceding stimulus: Knight \& Mather, 2009; Sakaki et al., 2014, but see Clewett et al., 2017). The retrograde effects are not as clear when positive deviants are used, with both amnesic (Sakaki et al., 2014) and hypermnesic effects (Hurlemann et al., 2005, 2007a, 2007b) having been found.

The third generalization is that memory for standard stimuli following emotional (i.e., negative and positive) deviants is generally slightly impaired (i.e., anterograde amnesic effects: e.g., Strange et al., 2003; Hurlemann et al., 2005), although this effect does not always reach statistical significance (Strange et al., 2010).

Fourth, these retrograde as well as anterograde effects could be altered by drug administration (for reviews see: Hurlemann, 2008; Mather et al., 2016). While substances that reduce normal amygdala responses via blocking central norepinephrine (NE) release (e.g., propranolol hydrochloride; Clewett et al., 2017; Hurlemann et al., 2005; Strange, Hurlemann, \& Dolan, 2003) lead to an abolishment of all emotion-induced memory effects, substances that enhance NE release in the brain (reboxetine; Hurlemann et al., 2005) exaggerate these effects. It is assumed that these drugs alter retrograde memory effects by influencing the experienced arousal in response to the presentation of emotional deviant stimuli (Hurlemann, et al., 2005). Further, retrograde and anterograde emotion-induced memory effects seem to rely on a normally functioning amygdala (Strange et al., 2003; Hurlemann et al., 2007b).

Figures 2 and 3 illustrate retrograde and anterograde effects separately for negative and positive deviants. The data used for these figures stem from immediate free-recall tasks (for the ease of comparison) that employed picture-label pairs (six experiments) or words (six experiments). Since these experiments did not involve any prioritization condition and did not involve a manipulation of semantic relatedness, the reader can get a rough overview of the "pure" impact of emotional deviants on the recall rates of neighboring standard information (for an overview see Table 3).

In the first graph (Fig. 2), recall rates for the respective deviant emotion and position relative to the deviant are compared with the average standard recall reported in the respective experiments ("compare with standards method"; see above). Visual inspection indicates a ceiling effect for the recall of deviants in the EOP experiments that used picturelabel pairs. In these experiments, clear retrograde amnesic effects - around 20 percentage points (p.p.) lower recall as compared with standard recall - for stimuli preceding negative deviants and retrograde hypermnesic effects (5-10 p.p. higher recall) for stimuli preceding positive deviants are reported. Recall for neutral standards surrounding neutral deviants was somewhat lower than standard recall. Clear anterograde amnesic effects are visible for both negative and positive deviant picture-label pairs. In the case of words as stimulus material, retrograde amnesic effects of negative deviants were less pronounced (about 10 p.p. on average) as compared with picture-label pairs. In addition, oddball effects of negative deviants (on average about 18 p.p. higher recall) and neutral deviants (recall rates about 11 p.p. higher than standard recall) were not as clear as in picture-label pairs. Recall for standards following both negative and neutral deviant words did not, on average, differ from standard recall. Note that there were no reports of the effects of positive deviants available in any identified EOP experiment with words.

In the second graph (Fig. 3), the "contrastive method" is used to calculate emotion-induced memory effects. Values indicate the deviation of one emotion/position condition from the respective neutral condition (e.g., in order to calculate the value of the recall for standards preceding negative deviants, one has to subtract the recall for standards preceding neutral deviants from it).

Table 3 Overview of immediate free-recall results (Figs. 2 and 3)

\begin{tabular}{ll}
\hline & Recall probability \\
\hline Picture-label pairs & $\mathrm{Neg}-1<\mathrm{Neu}-1<\mathrm{S}<\mathrm{Pos}-1$ \\
& $\mathrm{~S}<\mathrm{Neg}=\mathrm{Pos}=\mathrm{Neu}$ \\
& $\mathrm{Neg}+1=\mathrm{Pos}+1<\mathrm{Neu}+1<\mathrm{S}$ \\
Words & $\mathrm{Neg}-1<\mathrm{S}=\mathrm{Neu}-1$ \\
& $\mathrm{~S}<\mathrm{Neu}<\mathrm{Neg}$ \\
& $\mathrm{S}=\mathrm{Neg}+1=\mathrm{Neu}+1$
\end{tabular}

Neg negative deviant, Pos positive deviant, Neu neutral deviant, -1 stimulus preceding a deviant, +1 stimulus following a deviant, $S$ standard 
In general, it seems that in experiments involving pictures, standard stimuli neighboring neutral deviants are remembered less well than neutral standard stimuli not neighboring a deviant. Thus, slight differences can emerge in the magnitude of detrimental and beneficial effects of emotional deviants depending on the source of comparison. Noteworthy, this difference did not emerge in EOPs with words.

Performance in delayed-memory tasks Knight and Mather (2009, Exp. 1) showed that neutral standard stimuli that were presented in sequences that include a negative deviant were more likely to be recognized in a delayed test 1 week after the immediate recall test session than neutral stimuli that had been presented in sequences with neutral deviants in the first session. In Experiment 2 they found that if participants were assigned to a "recall" condition (that involved an immediate recall test at the end of each trial), delayed recognition after a 1 -week delay was better for items preceding a negative deviant than items preceding a neutral deviant. This difference was not present in the "no-recall" condition, which did not demand an immediate recall test in session one.

In a series of EOPs, Strange et al. (2010) found that the presentation of human faces during the retrieval phase of cued recall tasks led to negative-emotion-induced retrograde amnesia. This effect only occurred if the recall task with interspersed faces was not conducted on the same day as the cued recall task without faces but on the following day.

The two studies involving delayed-memory tasks are rather hard to compare because of the different methods used. While the Knight and Mather study (2009) involves delayed recognition tasks with the emotion-induction taking place in session one, the experiments by Strange et al. (2010) include delayed cued recall tasks, in which the time point of emotion-induction is varied across the experiments. However, what the results do have in common is that the reported emotion-induced memory effects are time-dependent. The advantage for neutral standards preceding a deviant, which received a high attentional weight at session one, is only apparent in the delayed recognition task 1 week later (Knight \& Mather, 2009). Similarly, retrograde amnesic effects in the Strange et al. (2010) experiments needed at least 1 day of retention time to become observed.

\section{Theoretical background of amnesic/hypermnesic effects of emotional deviant stimuli}

Why and how do stimuli that stick out of the mass due to their emotionality influence the memory performance for temporally adjacent neutral stimuli? In this section, we provide an outline of some theories on emotion and memory that might explain, in part, the above-summarized emotion-induced memory effects in the EOP. We start with the arousal-biased competition theory that poses that all stimuli presented in a sequence in the EOP compete for attentional resources and that there is an advantage for arousing deviants, which under certain circumstances can either impair or enhance memory for neighboring stimuli.

As there is evidence for qualitative differences in the memory rate for neutral standards preceding positive and negative deviants, an account involving emotional valence as a key factor for emotion-induced effects is also discussed.

Arousal-biased competition (ABC) At any given time, one is surrounded by an array of stimuli, which all compete for attentional and perceptual resources. That particular stimulus that has the highest priority at the given time and place will finally win the race for cognitive resources, i.e., its active mental representation in the human brain (Beck \& Kastner, 2009). In short, ABC theory predicts that if arousal is induced while such a competition between two or more stimuli is taking place, the priority of the most salient stimulus is enhanced even more (Mather \& Sutherland, 2011). Hence, this arousaldriven bias leads to "winner-take-more" and "loser-take-less" effects.

There is a lot of evidence for arousal affecting the chance of retrieval of neutral standard information neighboring deviants in EOP experiments (Mather \& Sutherland, 2011). The above repeatedly stated emotion-induced retrograde amnesic effect for neutral standard stimuli preceding emotionally negative deviant stimuli in a list of words can be described as dependent on the experienced arousal during presentation of the deviants. In experiments using word lists, neutral standard stimuli preceding emotional deviant stimuli usually do not indicate the occurrence of a deviant stimulus, and therefore according to the authors - do not have a high priority compared with the following deviant stimulus. In terms of $A B C$ theory, experienced arousal at encoding enhances the priority of deviants even more, making the preceding (neutral) standard stimulus, which is competing for cognitive resources, less likely to be recalled. By contrast, those stimuli surviving the encoding stage and being salient enough to be recalled in an immediate recall task, can be considered high priority items in the consolidation stage, where they share the mnemonic enhancement of emotional deviant stimuli, and in turn, will be more likely to be remembered in delayed-memory tasks as compared with items previously presented before emotionally neutral deviant stimuli (Anderson et al., 2006; Knight, \& Mather, 2009).

A further effect $\mathrm{ABC}$ can account for is the amplification of top-down goal relevance effects on memory through emotional arousal (for both negative and positive deviant stimuli; Sakaki et al., 2014). In this experiment, emotional arousal enhanced memory for preceding neutral standard stimuli when people prioritized those stimuli, but impaired memory for preceding standards when those were not prioritized. Thus, the occurrence of retrograde amnesia 
or hypermnesia was dependent on the interaction between arousal and priority. The recognition enhancement of preceding stimuli through priority was, thus, even higher if arousal was induced. In this study, the arousal effect was on the same level for negative and positive deviants (i.e., not dependent on valence). This is in line with ABC theory predictions, in that arousal induction (independent of stimulus valence) through emotional deviant stimuli further increased memory for high-priority stimuli. However, note that in their 2017 experiment Clewett and colleagues were not able to replicate effects of prioritization on neutral standards preceding a negative oddball, in that there was no enhancement of memory rates for these stimuli observed.

In terms of $\mathrm{ABC}$ theory, effects of semantic relatedness (e.g., Smith \& Beversdorf, 2008) between to-be-remembered neighboring standard stimuli and negative deviant stimuli can also be described as arousal-driven memory enhancements or decrements. In an EOP with a free-recall task, usually a participant's goal is to remember as many stimuli of a presented sequence as possible. Every stimulus in an otherwise semantically unrelated word list, which can assist in the encoding and/or retrieval of other stimuli by its semantic relationship to these stimuli, could be considered a more salient stimulus and receive higher priority as compared with stimuli, which are semantically unrelated to all the other stimuli of a sequence (and, thus, cannot serve as cue at the retrieval stage).

According to $\mathrm{ABC}$ theory, the priority of a given stimulus should be pushed more to the extreme by arousal induction (e.g., through presentation of a negative deviant stimulus), leading to a higher likelihood of recall for the standard stimulus presented directly before a deviant stimulus if it is semantically related to the deviant (and therefore has a higher priority for the participant), or to a lower likelihood of recall if that stimulus does not share a semantic link with the deviant stimulus (and therefore is of low priority).

Interestingly, in the experiment by Smith and Beversdorf (2008) this is partly the case. Neutral standard stimuli preceding negative deviant stimuli received a mnemonic enhancement if the two stimuli were semantically related. But there was no enhancement for neutral standard stimuli preceding negative deviant stimuli, if they were not semantically related. On the other hand, there was no significant decrease of recall rates observed for these semantically unrelated standard stimuli as compared to the recall rate for control stimuli in this study, which is not in line with $\mathrm{ABC}$ theory.

We can conclude that $\mathrm{ABC}$ theory fits well with many findings in EOP experiments, which report modulations of memory performances through the presentation of emotional deviant stimuli. Nonetheless, there is evidence that does not support ABC theory's predictions: Sakaki et al. (2014), for example, failed to show anterograde hypermnesic effects for prioritized neutral stimuli following negative deviants, and
Clewett et al. (2017) were not able to replicate retrograde hypermnesic effects for prioritized neutral stimuli preceding negative deviants.

Even more importantly, the theory struggles to explain retrograde hypermnesic effects of emotionally positive deviant stimuli that were reported in some studies (Hurlemann et al., 2005, 2007a, 2007b). Since in these experiments all stimuli were chosen to be semantically unrelated and there was no instruction to prioritize one of the items of the sequences of eight pictures, it seems hard to explain these obviously valence-driven effects in terms of semantic relatedness or goal relevance. Importantly, Hurlemann and colleagues reported that the positive and negative deviant stimuli in their experiments had been chosen to be equally arousing. Thus, the differential effects of positive and negative deviants cannot be described as arousal-dependent in these experiments.

Valence influences detailedness of memories Kensinger (2009) reviewed evidence of valence-driven effects on episodic memory. She argued that negative events are remembered more accurately with regard to their details than are positive events. Importantly, this does not mean that the occurrence of negative events is more likely remembered than the occurrence of positive events. Rather, people tend to recall more information consistently in cases of negative events. For example, fans of a defeated baseball team revealed a higher consistency of memories of the championship final than did fans of the winning team (Kensinger, \& Schacter, 2006). Both fans of the winning team (positive emotion group) and fans of the losing team (negative emotion group), as well as spectators who were not particularly interested in baseball (neutral group), were asked to recall as much information on the event itself and on the personal context (as to where they watched the match, with whom, etc.). This recall test was conducted 6 days after the championship final with a subsequent memory test after 23-27 weeks. While the absolute amount of all recalled information did not differ between the negative and positive groups (but was higher than in the neutral group), the amount of event-related information provided by the participants was higher in the negative than in the positive group. In addition, participants in the negative group revealed more consistent memories (i.e., the quality of information provided did not change as much between test one and test two) as compared with the positive and the neutral group. However, participants in the positive group showed greater self-reported confidence in their memories than participants in the other groups.

With regard to the identified EOP literature on memory effects, effects of retrograde and anterograde amnesia can be explained by this valence-dependent mechanism: While negative events/stimuli enhance the recall/recognition of detailed central information (at the cost of memory of peripheral 
information), positive events/stimuli do not influence the degree of recall/recognition of central information, and thus do not decrease recall/recognition rates for peripheral information. In the case of EOP experiments, stimuli presented just before or after an emotional deviant could be described as peripheral information. Hence, this valence-dependent mechanism can account for retrograde amnesia through presentation of a negative deviant stimulus. It furthermore explains the absence of such detrimental effects in cases of positive deviant stimuli (Hurlemann et al., 2005, 2007a, 2007b). However, this mechanism cannot account for the additional enhancement of recall probability (i.e., the hypermnesic effect) of positive deviant stimuli found in these studies.

The role of experimental details Beside theoretical considerations that involve the impact of emotional valence and arousal on attention and/or memory processes, one could discuss influences that stem from the specific experimental designs in which retrograde and anterograde effects of emotional deviants can be observed.

What makes an oddball odd? When talking about the influence of emotional deviants on memory rates of neighboring standard information, it is easily assumed that this influence originates in the emotional deviance from the otherwise more homogenous stream of emotionally neutral standard stimuli. However, in many EOP experiments, it does not seem to be clear whether deviance in terms of emotional valence and/or arousal is the (only) driving factor of possible retrograde and anterograde influences on memory.

Strange et al. (2000) showed that emotionally negative deviant nouns led to an oddball effect (i.e., enhanced recall) when compared with the recall of semantically related neutral standard nouns, irrespective of depth of encoding (shallow or deep encoding task). Perceptual deviants (emotionally neutral words written in a different font) also led to an oddball effect, but only under shallow encoding instructions. Recall of semantic deviants, however, did not differ from standard recall under shallow encoding and was even clearly decreased when a deep encoding task was used.

A similar pattern of results was obtained by Strange et al. (2003), with perceptual deviants leading to oddball effects that were limited to shallow encoding conditions, and oddball effects for negative deviants under both shallow and deep encoding. In a similar study (Strange et al., 2008), oddball effects for negative deviant words and perceptual deviant words could be replicated. However, in this study there was no recall advantage for the negative as compared with the perceptual deviant words.

In summary, EOP experiments with words highlight the importance of depth of encoding with regard to possible oddball effects. In EOPs investigating only the memory performance for deviant stimuli, it is shown that the magnitude of oddball effects of negative deviants and perceptual deviants does not differ if shallow encoding tasks are employed (Strange et al., 2000, 2004). By contrast, if participants are required to engage in deep encoding tasks, no oddball effects are reported for perceptual deviants while oddball effects for negative deviants remain stable (Strange et al., 2003; Kroes, Strange, \& Dolan, 2010). This may imply that perceptual deviants lose their distinctiveness if the meaning of a given stimulus is more (encoding) task-relevant than its appearance (e.g., its font). Another possible explanation would be that the encoding advantage for deeply encoded neutral stimuli might simply reach the advantage of being perceptually distinct without having an additional impact on recall chance.

In another study that focused on oddball effects, Kamp et al. (2015) found that positive deviant words in sequences with neutral words did not lead to an enhanced recall of positive words, but negative deviants clearly did evoke an oddball effect when interspersed in sequences of neutral standards. To our knowledge, no study has yet shown retrograde and anterograde memory effects induced by emotionally positive words. The results by Kamp and colleagues may imply that positive words are not distinct enough to be recalled better than neutral standards and, thus, do not have the power to influence recall rates for neighboring stimuli. Note that Kamp et al. (2015) chose positive and negative deviants such that they were equally arousing. In the reviewed studies that employed pictorial material, however, (additional) distinctiveness was always achieved for all deviant types by differences in perceptual quality between standards and deviants. This might explain why in studies that used positive images as deviant stimuli, emotion-induced memory effects on neighboring stimuli are reported (e.g., Hurlemann et al., 2005), while there are to date no EOP experiments published that show these effects induced by emotionally positive deviant words.

In EOPs in which pictures were used there is a potential confound of perceptual deviance and emotional deviance. Here, deviants differ from each other in terms of emotional valence and/or arousal, but pictures used as standard stimuli also differ from all deviant stimuli in perceptual quality (though to varying degrees with regard to the different studies). Given that deviants and standards often differ clearly in terms of perceptual details, it is not surprising that oddball effects occur in all EOP studies with pictures. Also, since ceiling effects were found for all types of deviants in these studies (Hurlemann et al., 2005, 2007a, 2007b; Knight, \& Mather, 2009), no significant differences in recall rates between emotional and neutral deviants emerged.

Taken together, in EOPs with pictures, it remains rather unclear whether it is sufficient to insert an emotional deviant to induce emotion-dependent effects on neighboring standard information, or whether the emotional deviant needs to be made more salient - adding perceptual deviance - in order to obtain the numerously reported emotion-induced effects. 
Stimulus onset asynchrony (SOA) Facing emotion-induced anterograde effects, we can summarize existing evidence as follows: They are either non-significant (Strange et al., 2003, 2008; Miu et al., 2005) or of an amnesic nature (Clewett et al., 2017; Hurlemann et al., 2005, 2007a, 2007b; Knight \& Mather, 2009; Müller et al., 2009; Sakaki, Fryer, \& Mather, 2014; Smith \& Beversdorf, 2008), mostly irrespective of deviant stimulus valence.

Strange et al. (2010) indicated that anterograde effects of emotional deviants on neutral standard stimuli might be SOAdependent. The authors refer to EOP experiments with an SOA of 3,000 ms that did not reveal anterograde effects (Strange, Hurlemann, \& Dolan, 2003; Miu et al., 2005) as well as to EOPs with an SOA of 5,000 ms (Hurlemann et al., 2005, 2007a, 2007b) that, by contrast, did show detrimental anterograde effects of both negative and positive deviants. However, in their own experiments (Strange et al., 2010), SOAs of $4,000 \mathrm{~ms}$ were used, which led to numerically decreased recall of standards following emotional deviants, but not to a statistically significant decrease. Therefore, they might have tapped the "SOA border," and it might be that SOAs longer than $4 \mathrm{~s}$ produce anterograde effects reliably.

In fact, a SOA-dependency of anterograde effects is plausible since a possible impact by the attention-drawing deviant stimulus on the encoding quality of the following neutral stimulus is likely to decay over time. This could mean that a sufficiently long SOA could prevent significant anterograde effects.

However, anterograde effects are not (solely) dependent on SOA, since all identified EOPs with picture-label pairs (using SOAs between 1,000 and 5,000 ms) elicited significant amnesic anterograde effects for both positive and negative deviants. For EOP experiments with written words, the findings seem to be somewhat incoherent. Significant amnesic anterograde effects were found in some participants in the immediate free-recall conditions (for shallow and deep encoding) in the study by Miu et al. (2005). In this experiment, an SOA of 4,000 ms was used. Also, the EOP experiment by Smith and Beversdorf (2008), who used an SOA of 2,000 ms, produced significant amnesic anterograde effects. By contrast, in the rest of EOP experiments with words as stimuli, no significant anterograde amnesic effects were observed (Strange et al., 2010; Strange, Hurlemann, \& Dolan, 2003). In these cases, SOAs were 4,000 and $3,000 \mathrm{~ms}$, respectively.

In order to shed light on possible critical SOAs, we turn to a paradigm that is similar to the EOP. Schmidt and Schmidt (2016) investigated anterograde "carryover" effects using the so-called "timeout procedure." Here, only three words were presented sequentially: one test word - either emotional or non-emotional - preceded and followed by one neutral word each. After the presentation of these words, participants engaged in a short non-emotional distracter task until the next trial started. At the end of the session, participants took part in an old/new recognition task. In a series of six experiments, the authors found that anterograde amnesic effects only occurred if SOAs shorter than 2,000 ms were used. This could be interpreted as further evidence for different SOA borders with regard to anterograde effects when presenting pictures as compared with written words.

One important thing to consider when using pictorial stimuli in memory experiments is the picture superiority effect (e.g., Madigan, 1983; Paivio, 1969). It states that due to their visual detailedness, pictures facilitate visual imagery more than words do. Visual imagery, in turn, enhances the retention of material, but might also capture attention in an EOP because of its more elaborate rehearsal - thus leading to a decrease of memory for following neutral standards. Due to the visual complexity of the deviant pictures used in the identified EOP experiments, it is plausible to assume that emotioninduced anterograde effects extend to a rather long time interval (up to $5 \mathrm{~s}$ in the Hurlemann studies).

By contrast, reading is a highly automated task in adults and therefore the attentional capture through emotional deviant words should be much smaller. Maybe, in the EOP experiments with words that have been conducted so far, the detrimental effects of emotional words had already vanished when the next neutral standard words were presented. Thus, future EOP experiments on memory with rather short SOAs $(<2,000$ $\mathrm{ms}$ ) could test this hypothesis.

In addition, emotional pictures are shown to elicit a higher level of arousal than emotional words do (e.g., Hinojosa, Carretié, Valcárcel, Méndez-Bértolo, \& Pozo, 2009). Given the important role of arousal in memory experiments, with low-arousing stimuli being less likely recalled than higharousing stimuli (Bradley, Greenwald, Petry, \& Lang, 1992), it is not surprising that emotion-induced retrograde and anterograde effects are less pronounced in the reviewed EOPs with words as compared with EOPs involving pictures.

\section{Conclusions}

The EOP allows researchers to investigate memory for emotional material deviating from surrounding stimuli as well as how those emotional deviants influence memory for the surrounding stimuli. We reviewed evidence of 29 recent EOP experiments and discussed potential mechanisms by which the observed emotion-induced memory effects are evoked.

\section{Emotion-induced effects are arousal-dependent}

One of the main mechanisms by which emotional deviants influence the memory rate of temporally adjacent neutral standards is arousal induction. Negative deviants, which usually are highly arousing stimuli, typically lead to retrograde amnesia in immediate recall and recognition memory tests (e.g., 
Hurlemann et al., 2005; Müller et al., 2009; Strange et al., 2003). Additionally, in most EOP experiments, both negative and positive arousing deviants lead to a numerical decrease of the memory rate for neutral stimuli following the deviants. This anterograde amnesic effect sometimes reaches significance (e.g., Hurlemann et al., 2005; Knight \& Mather, 2009; Müller et al., 2009).

Highly arousing stimuli are shown to lead to strong amygdala activations at encoding that correlate with successful retrieval (e.g., Kensinger \& Corkin, 2004; Strange et al., 2004). According to the $\mathrm{ABC}$ theory, arousal induction can enhance the saliency of already salient stimuli that compete with other stimuli for cognitive resources. This mechanism can explain retrograde amnesia since it leads to a "winner-takes-more" effect allocating even more resources to the already salient/ distinct deviant stimulus in a sequence of neutral standards. However, experiments have shown that the stimuli surrounding negative deviants can also profit from arousal induction if they are especially task-relevant (Knight \& Mather, 2009; Sakaki et al., 2014). Making a neutral standard preceding a negative deviant more goal-relevant than the deviant itself usually resulted in retrograde hypermnesia.

However, emotional arousal is not sufficient to explain emotion-induced effects in every instance. Three EOP studies have shown that the presentation of positive deviants in a stream of neutral standard information may result in retrograde hypermnesia rather than amnesia.

\section{Emotion-induced effects are valence-dependent}

Hurlemann et al. (2005, 2007a, 2007b) found in multiple experiments that presenting healthy participants with emotionally positive picture-label pairs in a sequence of otherwise emotionally neutral standards leads to an enhanced recall rate for stimuli directly preceding the deviants. This retrograde hypermnesia is in stark contrast to the retrograde amnesia they found when using negative deviants in the same experiments. It is important to note that neither goal relevance nor semantic relatedness was manipulated in these experiments.

We believe that this arousal/valence interaction found in the Hurlemann studies needs further testing in order to answer questions like: how robust is this finding (especially with regard to the retrograde hypermnesic effects of positive deviants $)^{2}$, are these hypermnesic effects susceptible to change of certain experimental details, and - if proven reliable - why do positive deviants have a facilitating effect on the memory for

\footnotetext{
${ }^{2}$ In the reviewed EOP experiments, there are no effect sizes for the relevant contrasts between the neutral condition and the emotional condition(s) reported. In only a small minority of studies, $n=4$, do the reported statistics allow for the calculation of effect sizes. This small number of effect sizes is not very informative, and is likely to be inflated (e.g., Ioannidis, 2008). We highly encourage more research on emotion-induced memory effects in order to gain a more precise estimate of the true effect size by future (meta) analyses.
}

preceding neutral standards, while negative deviants decrease the probability of remembering preceding standards correctly? As, to our knowledge, there is no theory to date that would predict retrograde hypermnesic effects of positive deviants and retrograde amnesic effects of negative deviants in the EOP, more research on the topic is necessary for a better understanding of these emotion-induced memory effects.

\section{Future directions}

To date, the EOP has been successfully used to show that emotional processing has broader influences on memory. Future researchers may use our summary of the results and theoretical explanations, as well as our specific suggestions, to make further use of this paradigm and to determine the specifics of how emotional stimuli influence memory, what the roles of valence and arousal are, and why there are apparent contradictory findings in the literature.

In the following, we identify three important issues around these effects that should be considered and investigated in future research.

\section{The interplay of valence and arousal}

We are still far from understanding the specific role of arousal in the EOP on memory. Is arousal induction a prerequisite for emotion-induced memory effects? Maybe it could be beneficial to choose emotional and nonemotional deviants that do not differ much in terms of arousal. Of course, the identification of emotionally neutral stimuli that are as arousing as, for instance, emotionally negative stimuli is a hard task. Nonetheless, it has been accomplished in some experiments already (e.g., using words: Bayer, Sommer, \& Schacht, 2012; using pictures: Briggs \& Martin, 2009). Notably, it is important that these neutral arousing stimuli are not only rated emotionally neutral on average but are also neutral on a case-to-case basis. In order to achieve this, the standard deviation of the valence rating should be as small as possible.

Next to the issue of arousal, the cause for differential effects of emotional valence should be investigated in future studies. EOP experiments in which highly arousing positive deviants are used are rare. It should, for instance, be investigated whether retrograde hypermnesic effects of positive deviants extend from picture-label pairs to pure verbal material. We identified only a single EOP experiment that utilized verbal material and positive deviants (Miu et al., 2005). In this study, however, there was no reporting of analyses broken down by the valence of the emotional deviant. Based on the studies with picture-label pairs we would expect similar retrograde 
hypermnesic effects with verbal material. If this is not the case with verbal material alone, it raises questions about what differs between the processing of verbal and visual information. There are studies indicating that a retrograde hypermnesia through positive deviant words - if observed - should be expected to be smaller than effects evoked by positive deviant pictures, because of emotional words leading to a lower arousal level than emotional pictures (Hinojosa et al., 2009) and positive deviant words in a sequence of neutral words not leading to an oddball effect (Kamp et al., 2015).

It could also be fruitful to try to replicate the important finding of the retrograde hypermnesic effects reported by Hurlemann et al. (2005, 2007a, 2007b). If it turned out that they can be reliably produced in the EOP, their existence would raise even more interesting questions, such as: What are the mechanisms that lead to an enhancement of memory performance for stimuli that directly precede a positive event? In a study by Gable and Harmon-Jones (2010), it was found that positive affect may have differential effects on memory for central and peripheral information depending on the associated approach motivation. While positive affects high in approach motivation often occur in the context of high goal relevance, positive affects low in approach motivation occur after a goal has been reached or in situations when there is no goal. Gable and Harmon-Jones (2010; Exp. 2) presented their participants on each trial with a cue (either a neutral picture or an appetitive picture known to activate high approach motivation). A word was displayed next either in the center of the screen or in the periphery, which was followed by a flanker task. Participants were told to perform as well as possible on the flanker task and that the words were irrelevant to their task. Results of a recognition task at the end of the experiment showed that centrally presented words after appetitive pictures were recognized better than centrally presented words after neutral pictures. For peripherally presented words, the result pattern was the opposite, with better recognition after neutral pictures than after appetitive pictures.

One could speculate that in the Hurlemann experiments, which show retrograde hypermnesic effects, the positive deviants used led to a low approach motivational affect in the participants, which in turn increased their memory rate for neutral standards preceding the positive deviants. Note however, that the broadening of attention through "low approach stimuli" apparently did not extend to the stimuli following the positive deviants because - similar to trials in which negative deviants were presented - anterograde amnesic effects were reported (Hurlemann et al., 2005, 2007a, 2007b). In addition, effects of low- and high-approach motivation are not only found for emotionally positive but also negative stimuli (for a review, see Harmon-Jones, Harmon-Jones, \& Price, 2013). Thus, it could be worthwhile to carefully address the role of low- and high-approach motivational affect in future EOP experiments on memory.

\section{The role of distinctiveness}

By calling influences of emotional deviant stimuli "emotioninduced memory effects," it is directly implied that the emotional nature (and thus, arousal and valence) play a crucial role in the manifestation of the effects. It is, however, important to note that there is a potential confound between perceptual and emotional distinctiveness of deviants in many of the reviewed experiments. In all cases of experiments with pictorial material, emotional deviants have differed from standards not only in terms of emotional content (i.e., arousal and valence) but also in perceptual details.

Future experiments that aim at better controlling this aspect will allow for stronger conclusions based on the emotional content of deviant stimuli. It could be the case that a certain perceptual dissimilarity, in EOPs with pictorial material, is a prerequisite for the relatively large emotion-induced effects (if compared to EOPs with words). A sensible approach would be to investigate emotion-induced effects in tasks that employ non-emotional standards as well as emotional and semantic deviants, while standards and deviants should not differ much from each other with regard to their physical properties.

Additionally, contrasting emotional standards and emotional deviants (as done in other EOP studies; for a review see Schlüter \& Bermeitinger, 2017) could lead to a better understanding of the nature of emotion-induced memory effects.

\section{The time course of emotion-induced effects}

The time course of emotion-induced effects has received little attention to this point. In one study (Anderson et al., 2006), non-emotional stimuli were remembered better when presented before an emotional than a non-emotional stimulus, but only in the short SOA condition ( $4 \mathrm{~s}$ rather than $9 \mathrm{~s}$ ). This issue is potentially even more important when considering anterograde memory effects, which presumably reflect attentional capture by emotional deviant stimuli. A related question is whether this time course is fixed, or is related to other factors, such as the arousal level of the deviant stimulus. Future experiments that specifically manipulate the SOA will provide us with critical information as to the time course in which emotional stimuli influence memory for surrounding neutral stimuli.

An issue related to the time course is the question of whether retrograde and anterograde emotion-induced effects are due to influences on the encoding or on the retrieval stage. While it seems most plausible that stimuli following an emotional deviant suffer from less efficient encoding because of the attention-drawing nature of the deviant, it is still necessary to test this hypothesis directly. In the case of retrograde effects of negative deviants, it is even less clear whether an impairment of memory performance for the standard stimulus 
preceding the deviant reflects an impaired stimulus rehearsal during encoding or whether the mental representation of the negative deviant at retrieval overshadows the representations of information that was presented close to the deviant in time. It is also possible that both mechanisms might play a (additive) role in emotion-induced effects. Future experiments should test for emotion-induced effects on encoding by varying presentation time or SOA, and influences on the stage of retrieval by investigating whether emotion-induced effects are coupled to a successful retrieval of the (emotional) deviants in immediate free-recall tasks.

\section{The EOP in memory research}

In sum, we conclude that the EOP still has much to offer for researchers interested in the intersection of emotion and memory. Not only does the paradigm allow for investigations into memory for emotional stimuli themselves, but also how the presentation of those stimuli influences memory for surrounding events. The EOP is intriguingly simple and the oddball paradigm is a well-established method. However, this should not belie some important issues regarding the experimental design that need careful consideration.

There has, for example, been little systemization in the past as to what the reference point is for considering amnesic and hypermnesic effects. Some studies have compared memory for standards preceding neutral deviants to memory for standards preceding emotional deviants (e.g., Clewett et al., 2017; Hurlemann et al., 2005, Sakaki et al., 2014), while others have compared memory for deviant-preceding standards to some measure of non-deviant-neighboring standards (e.g., Müller et al., 2009; Smith \& Beversdorf, 2008; Strange et al., 2003, 2008). For the sake of comparison, we propose that future experiments should not only include comparisons of emotion-induced effects with the effects of non-emotional deviants, but should also involve comparisons of these memory rates with memory performance for neutral standards not neighboring a deviant. This will allow for a more precise understanding of what we mean when we discuss relative amnesic and hypermnesic effects. It is important to note, however, that this may in some cases be limited by the method used in the particular experiment. For example, it is important to take into account potential primacy and recency effects. In experiments that use only a limited number of stimuli per list, this greatly reduces the number of comparison stimuli within each list. Therefore, it is of the utmost importance that future authors be as precise as possible in their descriptions of how comparison standard stimuli were chosen.

To the best of our knowledge, recent EOP experiments on memory have focused exclusively on visual presentation. This raises the question of whether these effects are modality specific or could be found in other modalities as well (e.g., auditory or olfactory). Not only would including other modalities provide the results with more external validity (since we live in a multi-modal world) but finding similar effects in other modalities (or in multi-modal experiments) would provide more evidence that the effects reflect a basic fact about how emotional processing (rather than, for example, visual perception) of a stimulus influences memory for that stimulus and neighboring stimuli. Furthermore, this could provide us with more information concerning the time course of emotional effects, as the perception of some stimuli (e.g., auditory) is considerably faster than the perception of other stimuli (e.g., olfactory).

\section{References}

Adelman, J. S., \& Estes, Z. (2013). Emotion and memory: A recognition advantage for positive and negative words independent of arousal. Cognition, 129(3), 530-535. doi:https://doi.org/10.1016/j.cognition. 2013.08.014

Anderson, A. K., Wais, P. E., \& Gabrieli, J. D. (2006). Emotion enhances remembrance of neutral events past. Proceedings of the National Academy of Sciences, 103(5), 1599-1604. doi:https://doi.org/10. 1073/pnas.0506308103

Bayer, M., Sommer, W., \& Schacht, A. (2012). P1 and beyond: Functional separation of multiple emotion effects in word recognition. Psychophysiology, 49(7), 959-969. doi:https://doi.org/10.1111/ j.1469-8986.2012.01381.x

Beck, D. M., \& Kastner, S. (2009). Top-down and bottom-up mechanisms in biasing competition in the human brain. Vision research, 49(10), 1154-1165. doi:https://doi.org/10.1016/j.visres.2008.07.012

Bradley, M. M., \& Lang, P. J. (1999). Affective norms for English words (ANEW): Instruction manual and affective ratings (Vol. 30, No. 1, pp. 25-36). Technical report C-1, the center for research in psychophysiology, University of Florida.

Bradley, M. M., Greenwald, M. K., Petry, M. C., \& Lang, P. J. (1992). Remembering pictures: pleasure and arousal in memory. Journal of experimental psychology: Learning, Memory, and Cognition, 18(2), 379. doi:https://doi.org/10.1037/0278-7393.18.2.379

Briggs, K. E. \& Martin, F. H. (2009). Affective picture processing and motivational relevance: arousal and valence effects on ERPs in an oddball task. International Journal of Psychophysiology, 72(3), 299-306. doi:https://doi.org/10.1016/j.ijpsycho.2009.01.009

Cahill, L., Prins, B., Weber, M., \& McGaugh, J. L. (1994). $\beta$-Adrenergic activation and memory for emotional events. Nature, 371(6499), 702-704. doi: $10.1038 / 371702 \mathrm{a} 0$

*Clewett, D., Sakaki, M., Nielsen, S., Petzinger, G., \& Mather, M. (2017). Noradrenergic mechanisms of arousal's bidirectional effects on episodic memory. Neurobiology of learning and memory, 137, 1-14. doi:https://doi.org/10.1016/j.nlm.2016.10.017

Detterman, D. K. (1976). The retrieval hypothesis as an explanation of induced retrograde amnesia. The Quarterly journal of experimental psychology, 28(4), 623-632. doi:10.1080/14640747608400588

Detterman, D. K. \& Ellis, N. R. (1972). Determinants of induced amnesia in short-term memory. Journal of Experimental Psychology, 95(2), 308-316. doi:10.1037/h0033629

Dolcos, F., LaBar, K. S., \& Cabeza, R. (2004). Dissociable effects of arousal and valence on prefrontal activity indexing emotional evaluation and subsequent memory: an event-related fMRI study. Neuroimage, 23(1), 64-74. doi:https://doi.org/10.1016/j. neuroimage.2004.05.015 
Ellis, N. R., Detterman, D. K., Runcie, D., McCarver, R. B., \& Craig, E. M. (1971). Amnesic effects in short-term memory. Journal of Experimental Psychology, 89(2), 357-361. doi:https://doi.org/10. 1037/h0031192

*Froeliger, B., Gilbert, D. G., \& McClernon, F. J. (2009). Effects of nicotine on novelty detection and memory recognition performance: Double-blind, placebo-controlled studies of smokers and nonsmokers. Psychopharmacology, 205, 625-633. doi:https://doi.org/ 10.1007/s00213-009-1571-y

Gable, P. A. \& Harmon-Jones, E. (2010). The effect of low versus high approach-motivated positive affect on memory for peripherally versus centrally presented information. Emotion, 10(4), 599-603. doi: https://doi.org/10.1037/a0018426

Hamann, S. (2001). Cognitive and neural mechanisms of emotional memory. Trends in cognitive sciences, 5(9), 394-400. doi:https:// doi.org/10.1016/S1364-6613(00)01707-1

Harmon-Jones, E., Harmon-Jones, C., \& Price, T. F. (2013). What is approach motivation? Emotion Review, 5(3), 291-295. doi:https:// doi.org/10.1177/1754073913477509

Hinojosa, J. A., Carretié, L., Valcárcel, M. A., Méndez-Bértolo, C., \& Pozo, M. A. (2009). Electrophysiological differences in the processing of affective information in words and pictures. Cognitive, Affective, \& Behavioral Neuroscience, 9(2), 173-189. doi:https:// doi.org/10.3758/CABN.9.2.173

Hurlemann, R. (2008). Noradrenergic-glucocorticoid mechanisms in emotion-induced amnesia: from adaptation to disease. Psychopharmacology, 197(1), 13-23. doi:https://doi.org/10.1007/ s00213-007-1002-x

*Hurlemann, R., Hawellek, B., Maier, W., \& Dolan, R. J. (2007a). Enhanced emotion-induced amnesia in borderline personality disorder. Psychological Medicine, 37, 971-981. doi:https://doi.org/10. 1017/S0033291706009792

*Hurlemann, R., Hawellek, B., Matusch, A., Kolsch, H., Wollersen, H., Madea, B., ... \& Dolan, R. J. (2005). Noradrenergic modulation of emotion-induced forgetting and remembering. The Journal of Neuroscience, 25(27), 6343-6349. doi:https://doi.org/10.1523/ JNEUROSCI.0228-05.2005

*Hurlemann, R., Wagner, M., Hawellek, B., Reich, H., Pieperhoff, P., Amunts, K., ... \& Dolan, R. J. (2007b). Amygdala control of emotion-induced forgetting and remembering: Evidence from Urbach-Wiethe disease. Neuropsychologia, 45, 877-884. doi: https://doi.org/10.1016/j.neuropsychologia.2006.08.027

Ioannidis, J. P. (2008). Why most discovered true associations are inflated. Epidemiology, 19(5), 640-648. doi:https://doi.org/10.1097/EDE. 0b013e31818131e7

*Kamp, S. M., Potts, G. F., \& Donchin, E. (2015). On the roles of distinctiveness and semantic expectancies in episodic encoding of emotional words. Psychophysiology, 52(12), 1599-1609. doi:https://doi. org/10.1111/psyp. 12537

Kensinger, E. A. (2009). Remembering the details: Effects of emotion. Emotion review, 1(2), 99-113. doi:https://doi.org/10.1177/ 1754073908100432

Kensinger, E. A., \& Corkin, S. (2004). Two routes to emotional memory: Distinct neural processes for valence and arousal. Proceedings of the National Academy of Sciences, 101(9), 3310-3315. doi:https://doi. org/10.1073/pnas.0306408101

Kensinger, E. A., Garoff-Eaton, R. J., \& Schacter, D. L. (2006). Memory for specific visual details can be enhanced by negative arousing content. Journal of Memory and Language, 54(1), 99-112. doi: https://doi.org/10.1016/j.jml.2005.05.005

Kensinger, E. A. \& Schacter, D. L. (2006). When the Red Sox shocked the Yankees: Comparing negative and positive memories. Psychonomic Bulletin \& Review, 13(5), 757-763. doi:https://doi. org/10.3758/BF03193993
*Knight, M. \& Mather, M. (2009). Reconciling findings of emotioninduced memory enhancement and impairment of preceding items. Emotion, 9(6), 763-781. doi:https://doi.org/10.1037/a0017281

*Kroes, M. C., Strange, B. A., \& Dolan, R. J. (2010). $\beta$-adrenergic blockade during memory retrieval in humans evokes a sustained reduction of declarative emotional memory enhancement. Journal of Neuroscience, 30(11), 3959-3963. doi:https://doi.org/10.1523/ JNEUROSCI.5469-09.2010

Lang, P. J., Bradley, M. M., \& Cuthbert, B. N. (1999). International affective picture system (IAPS): Instruction manual and affective ratings. Gainesville, FL: The Center for Research in Psychophysiology, University of Florida.

Lundqvist, D., Flykt, A., \& Öhman, A. (1998). The Karolinska Directed Emotional Faces - KDEF, CD ROM from Department of Clinical Neuroscience, Psychology section, Karolinska Institutet, ISBN 91630-7164-9.

Madigan, S. (1983). Picture memory. In J. Yuille (ed.), Imagery, memory, and cognition: Essays in honor of Allan Paivio (pp.65-89). New York (NY): Lawrence Erlbaum Associates.

Markovic, J., Anderson, A. K., \& Todd, R. M. (2014). Tuning to the significant: Neural and genetic processes underlying affective enhancement of visual perception and memory. Behavioural brain research, 259, 229-241. doi:https://doi.org/10.1016/j.bbr.2013.11. 018

Mather, M., \& Nesmith, K. (2008). Arousal-enhanced location memory for pictures. Journal of memory and language, 58(2), 449-464. doi: https://doi.org/10.1016/j.jml.2007.01.004

Mather, M. \& Sutherland, M. R. (2011). Arousal-biased competition in perception and memory. Perspectives on Psychological Science, 6(2), 114-133. doi:https://doi.org/10.1177/1745691611400234

Mather, M., Clewett, D., Sakaki, M., \& Harley, C. W. (2016). Norepinephrine ignites local hotspots of neuronal excitation: How arousal amplifies selectivity in perception and memory. Behavioral and Brain Sciences, 39. doi:https://doi.org/10.1017/ S0140525X15000667

*Miu, A. C., Heilman, R. M., Opre, A., \& Miclea, M. (2005). Emotioninduced retrograde amnesia and trait anxiety. Journal of Experimental Psychology: Learning, Memory, and Cognition, 31, 1250-1257. doi:https://doi.org/10.1037/0278-7393.31.6.1250

*Müller, N. G., Wohlrath, B., Kopp, U. A., \& Lengler, U. (2009). Emotional content does not interfere with verbal memory in patients with temporal lobe epilepsy. Epilepsy \& Behavior, 15(3), 367-371. doi:https://doi.org/10.1016/j.yebeh.2009.05.006

Ogden, C.K. (1932). Basic English: A general introduction with rules and grammar (3rd ed.). London: K. Paul, Trench, Trubner \& Co., Ltd.

Paivio, A. (1969). Mental imagery in associative learning and memory. Psychological review, 76(3), 241-263. doi:https://doi.org/10.1037/ h0027272

Richardson, M. P., Strange, B. A., \& Dolan, R. J. (2004a). Encoding of emotional memories depends on amygdala and hippocampus and their interactions. Nature neuroscience, 7(3), 278-285. doi:https:/ doi.org/10.1038/nn1190

Richardson, M. P., Strange, B. A., Duncan, J. S., \& Dolan, R. J. (2003). Preserved verbal memory function in left medial temporal pathology involves reorganisation of function to right medial temporal lobe. Neuroimage, 20, S112-S119. doi:https://doi.org/10.1016/j. neuroimage.2003.09.008

Richardson, M. P., Strange, B. A., Duncan, J. S., \& Dolan, R. J. (2006). Memory fMRI in left hippocampal sclerosis optimizing the approach to predicting postsurgical memory. Neurology, 66(5), 699705. doi:https://doi.org/10.1212/01.wnl.0000201186.07716.98

Richardson, M. P., Strange, B. A., Thompson, P. J., Baxendale, S. A., 1933 Duncan, J. S., \& Dolan, R. J. (2004b). Pre-operative verbal memory 1934 fMRI predicts post-operative memory decline after left temporal 1935 lobe resection. Brain, 127(11), 2419-2426. doi: https://doi.org/10.1093/brain/awh293 
Roediger III, H. L. (2008). Relativity of remembering: Why the laws of memory vanished. Annual Review of Psychology, 59, 225-254. doi: https://doi.org/10.1146/annurev.psych.57.102904.190139 Runcie, D., \& O'Bannon, R. M. (1977). An independence of induced amnesia and emotional response. The American journal of psychology, 55-61. http://www.jstor.org/stable/1421640

*Sakaki, M., Fryer, K., \& Mather, M. (2014). Emotion strengthens highpriority memory traces but weakens low-priority memory traces. Psychological Science, 25(2), 387-395. doi:https://doi.org/10.1177/ 0956797613504784

Saufley, W. H., Jr. \& Winograd, E. (1970). Retrograde amnesia and priority instructions in free recall. Journal of Experimental Psychology, 85(1), 150-152. doi:https://doi.org/10.1037/h0029538

Schlüter, H. \& Bermeitinger, C. (2017). Emotional oddball: A review on variants, results, and mechanisms. Review of General Psychology, 21(3), 179-222. doi:https://doi.org/10.1037/gpr0000120

Schmidt, S. R. (2002). Outstanding memories: The positive and negative effects of nudes on memory. Journal of Experimental Psychology: Learning, Memory, and Cognition, 28(2), 353-361. doi:https://doi. org/10.1037/0278-7393.28.2.353

Schmidt, S. R. \& Schmidt, C. R. (2016). The emotional carryover effect in memory for words. Memory, 24(7), 916-938. doi:https://doi.org/ $10.1080 / 09658211.2015 .1059859$

Schultz, L. S. (1971). Effects of high priority events on recall and recognition of other items. Journal of Verbal Learning and Verbal Behavior, 10, 322-330. doi:https://doi.org/10.1016/S00225371(71)80062-2

*Smith, R. M. \& Beversdorf, D. Q. (2008). Effects of semantic relatedness on recall of stimuli preceding emotional oddballs. Journal of the International Neuropsychological Society, 14, 620-628. doi: https://doi.org/10.1017/S1355617708080843

Snodgrass, J. G. \& Vanderwart, M. (1980). A standardized set of 260 pictures: norms for name agreement, image agreement, familiarity, and visual complexity. Journal of experimental psychology: Human learning and memory, 6(2), 174-215.

Strange, B. A. \& Dolan, R. J. (2001). Adaptive anterior hippocampal responses to oddball stimuli. Hippocampus, 11, 690-698. doi: https://doi.org/10.1002/hipo.1084

*Strange, B. A. \& Dolan, R. J. (2004). $\beta$-Adrenergic modulation of emotional memory-evoked human amygdala and hippocampal responses. Proceedings of the National Academy of Sciences of the United States of America, 101, 11454-11458. doi:https://doi.org/10. 1073/pnas.0404282101

Strange, B. A., \& Dolan, R. J. (2007). $\beta$-adrenergic modulation of oddball responses in humans. Behavioral and Brain Functions, 3-29. doi:https://doi.org/10.1186/1744-9081-3-29

Strange, B., \& Galarza-Vallejo, A. (2016). Bidirectional synaptic plasticity can explain bidirectional retrograde effects of emotion on memory. Behavioral and Brain Sciences, 39, E224. doi:https://doi. org/10.1017/S0140525X15001958

*Strange, B. A., Gartmann, N., Brenninkmeyer, J., Haaker, J., Reif, A., Kalisch, R., \& Büchel, C. (2014). Dopamine receptor 4 promoter polymorphism modulates memory and neuronal responses to salience. Neuroimage, 84, 922-931. doi:https://doi.org/10.1016/j. neuroimage.2013.09.065

*Strange, B. A., Henson, R. N. A., Friston, K. J., \& Dolan, R. J. (2000). Brain mechanisms for detecting perceptual, semantic, and emotional deviance. Neuroimage, 12(4), 425-433. doi:https://doi.org/10.1006/ nimg.2000.0637

*Strange, B. A., Hurlemann, R., \& Dolan, R. J. (2003). An emotioninduced retrograde amnesia in humans is amygdala- and $\beta$ adrenergic dependent. Proceedings of the National Academy of Sciences of the United States of America, 100, 13626-13631. doi: https://doi.org/10.1073/pnas.1635116100

*Strange, B. A., Kroes, M. C., Fan, J., \& Dolan, R. J. (2010). Emotion causes targeted forgetting of established memories. Frontiers in Behavioral Neuroscience, 4, 175. doi:https://doi.org/10.3389/ fnbeh.2010.00175

*Strange, B. A., Kroes, M. C., Roiser, J. P., Tan, G. C., \& Dolan, R. J. (2008). Emotion-induced retrograde amnesia is determined by a 5 HTT genetic polymorphism. Journal of Neuroscience, 28(28), 7036-7039. doi:https://doi.org/10.1523/JNEUROSCI.0834-08. 2008

Tarr, M. J. The object databank. 2005. http://www.tarrlab.org/

Tulving E. (1969). Retrograde amnesia in free recall. Science, 164, 8890. doi:https://doi.org/10.1126/science.164.3875.88

Von Restorff, H. (1933). Über die wirkung von bereichsbildungen im spurenfeld. Psychologische Forschung, 18(1), 299-342. doi:https:// doi.org/10.1007/BF02409636

Wallace, W. P. (1965). Review of the historical, empirical, and theoretical status of the Von Restorff phenomenon. Psychological bulletin, 63(6), 410-424. doi:https://doi.org/10.1037/h0022001

*West Saxvig, I., Johansen Lundervold, A., Grønli, J., Ursin, R., Bjorvatin, B., \& Portas, C. M. (2008). The effect of a REM sleep deprivation procedure on different aspects of memory function in humans. Psychophysiology, 45, 309-317. doi:https://doi.org/10. 1111/j.1469-8986.2007.00623.x

Wolfe, J. M. (2003) Moving towards solutions to some enduring controversies in visual search. Trends in Cognitive Sciences, 7, 70-76. doi: https://doi.org/10.1016/S1364-6613(02)00024-4

Publisher's note Springer Nature remains neutral with regard to jurisdictional claims in published maps and institutional affiliations. 